\title{
Isotopic signatures and hydrochemistry in assessing groundwater occurrence in the desert environs west Qena, Egypt
}

\author{
Mohamed Abdel Hadi Shedid El Sabri ${ }^{1}$, Mohamed Ahmed Rashed ${ }^{2}$, Kadry Nasser Sediek²,
} Nabil Hosny Rofaayl ${ }^{1}$ and Osama Hazem Eltabakh ${ }^{1 *}$ (e)

\begin{abstract}
Background: Developing the desert zone outside the flood plain of the Nile River and targeting the reclamation of about 12,500 feddan in the desert environs of Qena governorate are considered one of the top priorities of both government and the private sectors. The present study is based on qualitative analyses of the groundwater of the Quaternary aquifer in the desert environs west Qena. Groundwater was chemically and isotopically analyzed to determine the origin of groundwater and assess its occurrence.

Results: The obtained results indicate that the Quaternary aquifer represents the most important groundwater aquifer in the area. The water level ranges from +40 to $+67 \mathrm{~m}$ above sea level. The groundwater movement direction in the study area is mainly from southeast to northwest direction, with some local flow groundwater directions from Nile River at the north to old alluvial plain and desert environs at the south. The hydraulic conductivity of the aquifer is determined from the pumping test range from 17.63 to $42.86 \mathrm{~m} /$ day, with an average value of $27.04 \mathrm{~m} /$ day, while the transmissivity ranges between 2142.8 and $1128.3 \mathrm{~m}^{2} /$ day, with an average value of $1456.74 \mathrm{~m}^{2} /$ day. The total dissolved solids range from 589 to $3832 \mathrm{mg} / \mathrm{L}$. Isotopic analysis (oxygen eighteen and deuterium) of fifteen groundwater samples was carried out to define the recharge source of the Quaternary aquifer. The groundwater isotopic data reflect variability in recharge conditions during different ages and different climatic regimes. According to the minor and trace elements concentration and US Salinity Laboratory Staff nomogram, most water samples are suitable for drinking purposes, domestic use and irrigation.
\end{abstract}

Conclusion: The current research concluded that from the foregoing hydrological study and the determined values of hydraulic conductivity and transmissivity, the Quaternary aquifer is classified as ranging between moderate and high potential aquifer. The high potentiality of the aquifer is detected close to the Nile River. In addition to the representative groundwater samples showing the isotope signature of the Nile River sample, this means that all these wells have the same source of recharge (meteoric water of the Nile River).

Keywords: Hydro-geochemistry, Groundwater, Isotopes, Hydrology, Hydrogeology, Qena, Egypt

\footnotetext{
*Correspondence: o.h.eltabakh@gmail.com

1 Hydrology Department, Desert Research Center, P.O. Box 11753, El

Matariya, Cairo, Egypt

Full list of author information is available at the end of the article
}

\begin{abstract}
Background
In developing countries, increasing population constitutes a big problem because of its great effects on the national growth leading to the increase in poverty and social problems. The acquisition of new land for agriculture remains as one of the major solutions to minimize such harmful effects. In Egypt, there is a continuous
\end{abstract}


demand for more water necessary for reclamation of new land to be added to the existing cultivated land, so the development of groundwater resources in Egypt has received special attention a few years ago where the groundwater aquifers underlying the Nile Valley, the Delta, parts of the deserts and coastal area act as an auxiliary source of water in Egypt. Therefore, nowadays, the Egyptian Government and the private sector are interested in developing the desert zone outside the flood plain of the Nile River and targeting the reclamation of about 12,500 feddan in the west Qena area.

The study area is located in the southern part of Upper Egypt, bounded by latitudes $25^{\circ} 45^{\prime} 32.28^{\prime \prime}$ and $26^{\circ} 05^{\prime} 36.30^{\prime \prime} \mathrm{N}$ and longitudes $31^{\circ} 57^{\prime} 12.30^{\prime \prime}$ and $32^{\circ} 45^{\prime} 6.84^{\prime \prime} \mathrm{E}$ and covers a surface area about $2000 \mathrm{~km}^{2}$ (Fig. 1). Climatically, the study area is characterized by desert climatic conditions, dominated by long hot, rainless summer and arid warm winter. The annual mean

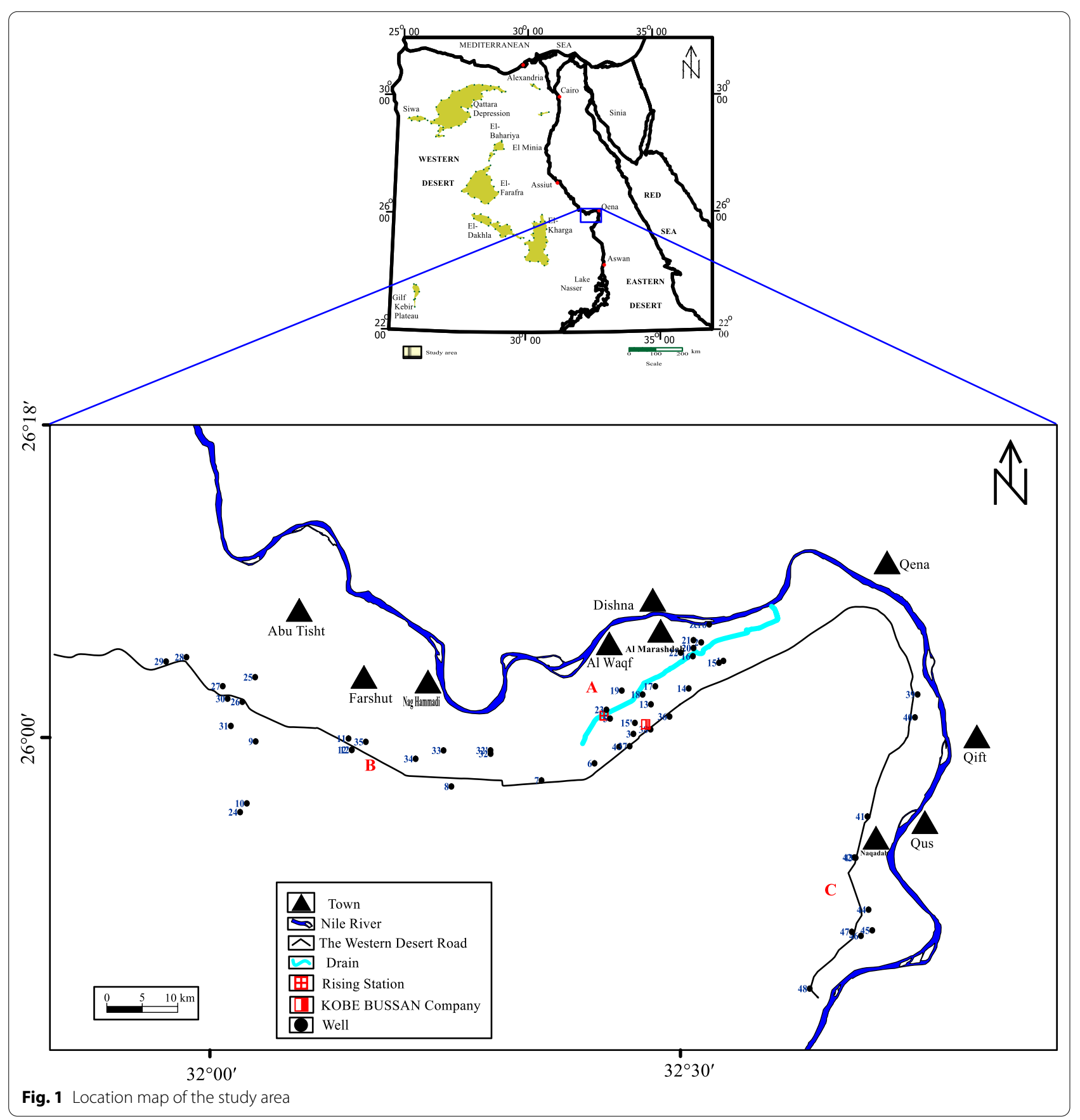


temperature is $31.5{ }^{\circ} \mathrm{C}$, the annual rainfall is $3.83 \mathrm{~mm}$, the annual mean of relative humidity is $30.68 \%$, and the annual mean value of wind speed is $8.73 \mathrm{~km} / \mathrm{h}$ (Tutiempo. net, 2018) and the intensity of evaporation is $11.28 \mathrm{~mm} /$ day (Mahmoud 2005).

The evaluation of groundwater resources for development requires an understanding of the hydrogeochemical properties of groundwater in the aquifer. The chemical characteristics of groundwater are mainly influenced by rock interaction, sources of recharge, direction and rate of groundwater movement in addition to other factors. Water chemistry plays an important role in hydrologic science, since it can be regarded as a tool revealing various hydrological processes in the past. Moreover, the occurrence of some ions and compounds in groundwater of a specific region may be used as indicators for the origin and formation of groundwater. This work aims to assess the chemical groundwater composition, isotopic signature and determine the origin of groundwater and the main recharge of the Quaternary aquifers in desert environs of the Qena area.
Geomorpholgically, the study area represents a portion along the western bank of the Nile River. The ground surface elevation decreases gradually from the southern limestone plateau to the Nile Valley plain. The giant deviation of the Nile River course, causing the Qena bend, was formed because of the intensive faulting (rift valley) and tectonic activities during different geologic ages. The area is subdivided into four geomorphic units (Said 1962). These are the young alluvial plains (the cultivated lands), the old alluvial plains (the Nile terraces which are a high level of the cultivated lands), the watershed area (the southern calcareous plateau) and water collectors' areas (alluvial fans and hydrographic basins) (Fig. 2).

Geologically, the sedimentary succession overlying the Precambrian basement rocks in west Qena area is belonging to the Paleozoic, the Upper Cretaceous, the Tertiary and Quaternary (CONOCO and EGPC 1987). A brief description of such succession is given in the following from older to younger (Fig. 3):

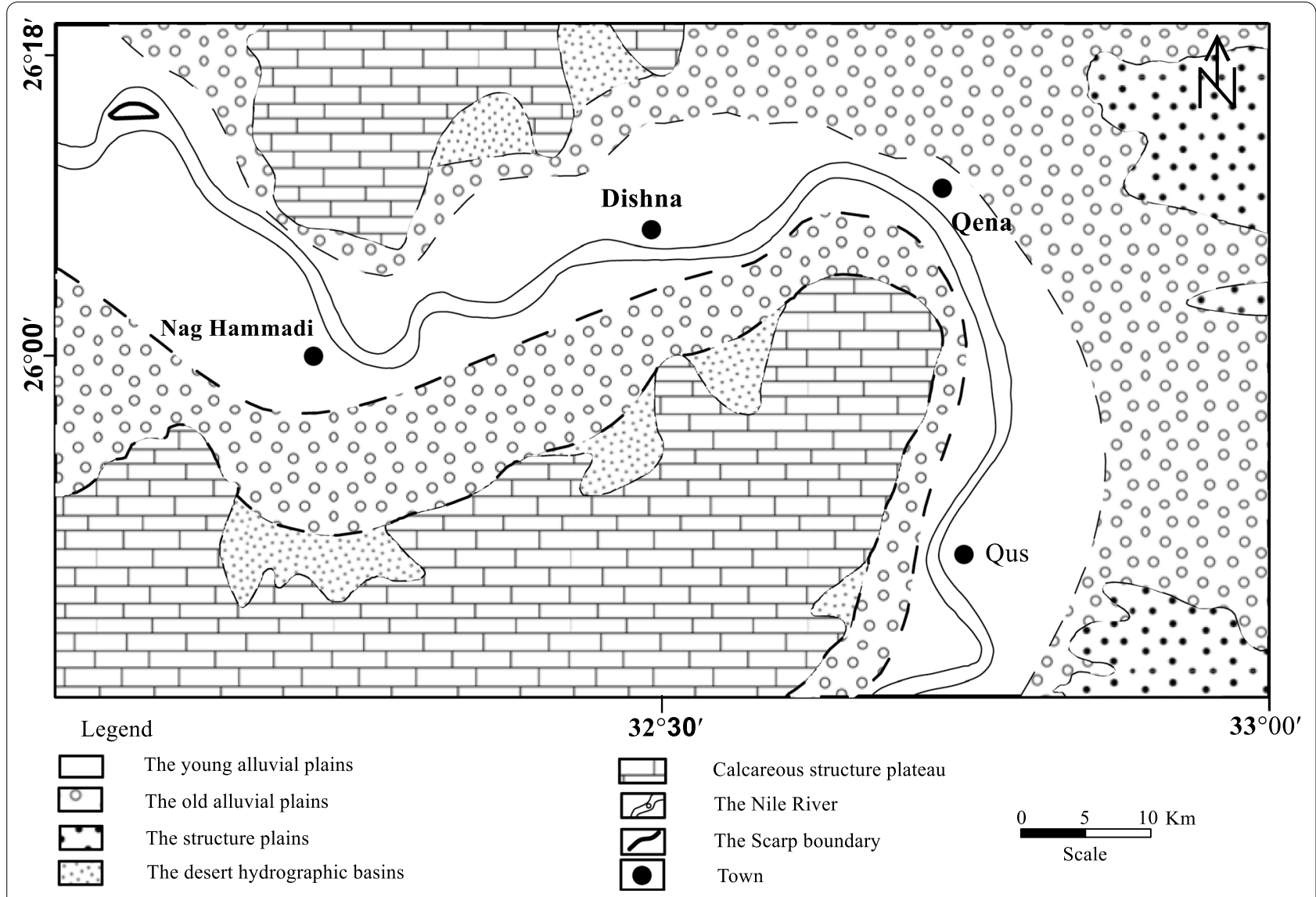

Fig. 2 Main geomorphologic units of the desert environs west Qena (Said 1962) 


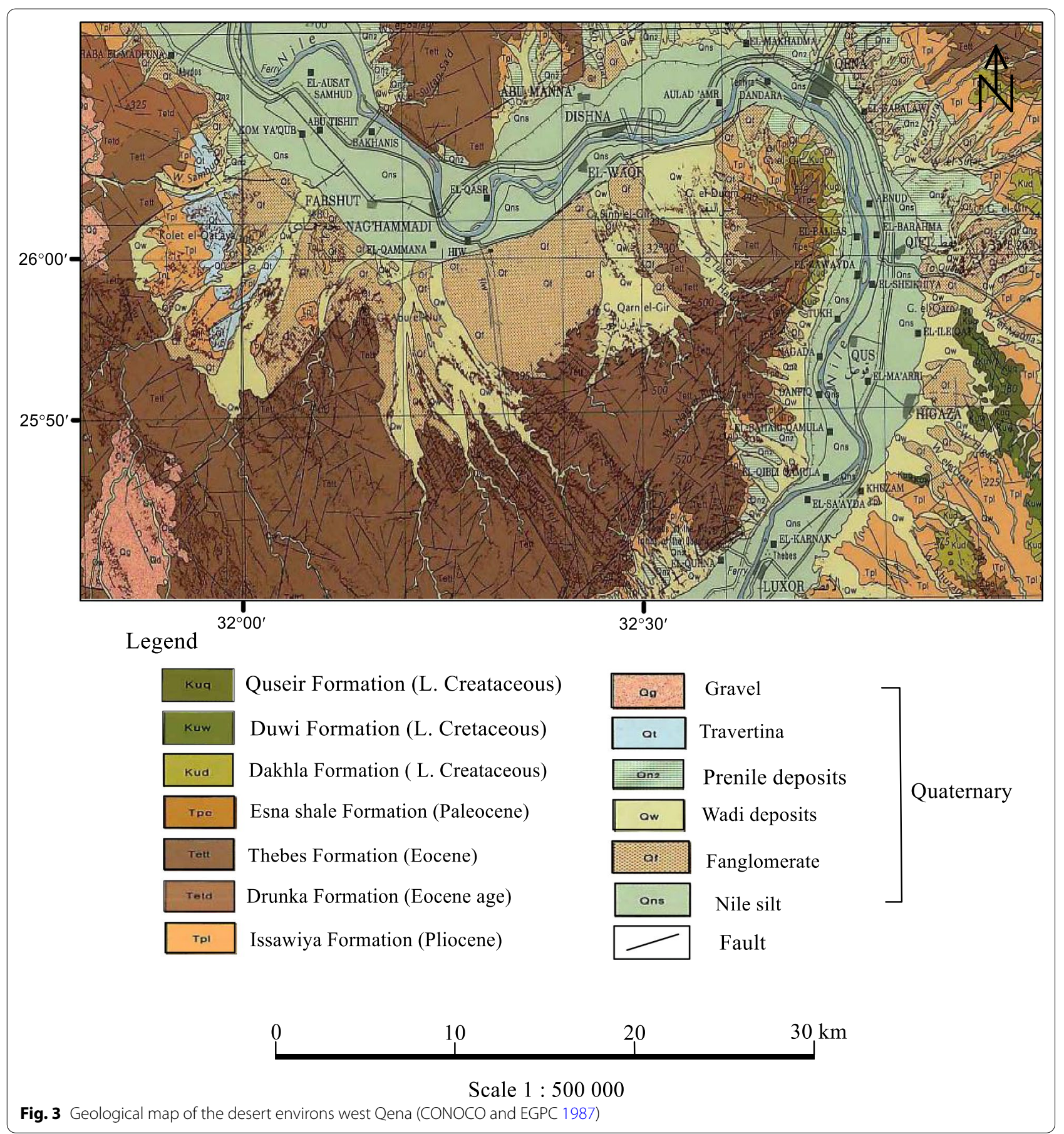

- Paleozoic-lower Cretaceous, which area composed of sandstone with intercalations of mudstone related to the Nubian Sandstone Formation.

- Upper Cretaceous, differentiated into Duwi Formation, which compose of phosphatic beds intercalated with shale and marl and Dakhla Formation that formed of shale with marl.
- Paleocene, differentiated into chalk and limestone of lower Tarawan Formation and shale of upper Esna Formation.

- Eocene, include chalk to chalky and dolomitic limestone of Thebes Formation.

- Pliocene, composed of interbedded clay and sand with silt of Madamud Formation. 
- Pleistocene, differentiated into three main deposition stages (paleonile/protonile, prenile and neonile), this succession composed of lower thick clay unit with silt and marl of Armant and Issawia Formation, the middle unit formed of massive sand with intercalated clay lenses and conglomerates of Qena Formation and the upper sandy silt unit with pebbles of Dandara Formation.

- Holocene, deposits are represented by Wadi fill deposits consisting of silty clay, sand and gravels.

Tectonically, the study area is slightly affected by tensional forces leading to several normal and wrench faults of NW-SE and NE-SW trends and mainly affecting the Eocene limestone. These faults are associated with some parallel folds (Fig. 4) (Said 1962 and Youssef 1968).

\section{Methods}

Fifty groundwater samples were collected from the Quaternary aquifer in addition to one surface water sample from Nile River for chemical analysis, also fifteen representative water samples were collected for isotopic analysis using plastic bottles, and each one was labeled with 6 number and highly sealed in December (2016). Direct measurements were made at each site in the field (in-situ measurements) giving readings for, total dissolved solids (TDS), electrical conductivity (EC), temperature and $\mathrm{pH}$ by using Combo $\mathrm{pH}$ and $\mathrm{EC}$ meter. The chemical analysis of these samples is carried out for major cations $\left(\mathrm{Ca}^{2+}\right.$, $\left.\mathrm{Mg}^{2+}, \mathrm{Na}^{+}, \mathrm{K}^{+}\right)$and some major anions $\left(\mathrm{Cl}-, \mathrm{SO}_{4}{ }^{2-}\right)$ by using ion chromatography (IC) in the water quality laboratory of the Desert Research Center (DRC), while carbonate $\left(\mathrm{CO}^{3-}\right)$ and bicarbonate $\left(\mathrm{HCO}^{3-}\right)$ were determined by titration. The analysis of some trace elements $\left(\mathrm{Si}^{4+}, \mathrm{B}^{3+}, \mathrm{Al}^{3+}, \mathrm{Li}+, \mathrm{Mo}, \mathrm{Sr}^{2+}, \mathrm{Ba}^{2+}, \mathrm{Cr}, \mathrm{Cu}, \mathrm{Fe}^{2+}, \mathrm{Mn}^{2+}\right.$, $\mathrm{Ni}, \mathrm{Pb}, \mathrm{V}$ and $\mathrm{Zn}$ ) for seventeen water sample was carried out by using inductively coupled plasma (ICP) in the Central Laboratory of the Desert Research Center (DRC). The isotopic analysis (measuring the isotopic ratios of $\mathrm{O}^{18}$ and $\mathrm{D}$ ) was carried out by (Hydrogeology Group of Freie Universitaet Berlin, Germany) using isotopes ratio mass spectrometer.

\section{Results}

The water-bearing sediments have a very wide geographical distribution in the Nile Valley and also in the adjacent desert Wadies. They are mainly composed of gravels, sands and clay, which relate to the Pliocene clay overlying the fissured carbonates form the base of the Quaternary aquifer in the area (Said 1981). The results are discussed under the following main topics; the hydrogeological aspect, the hydrogeochemical and isotopic characteristics.

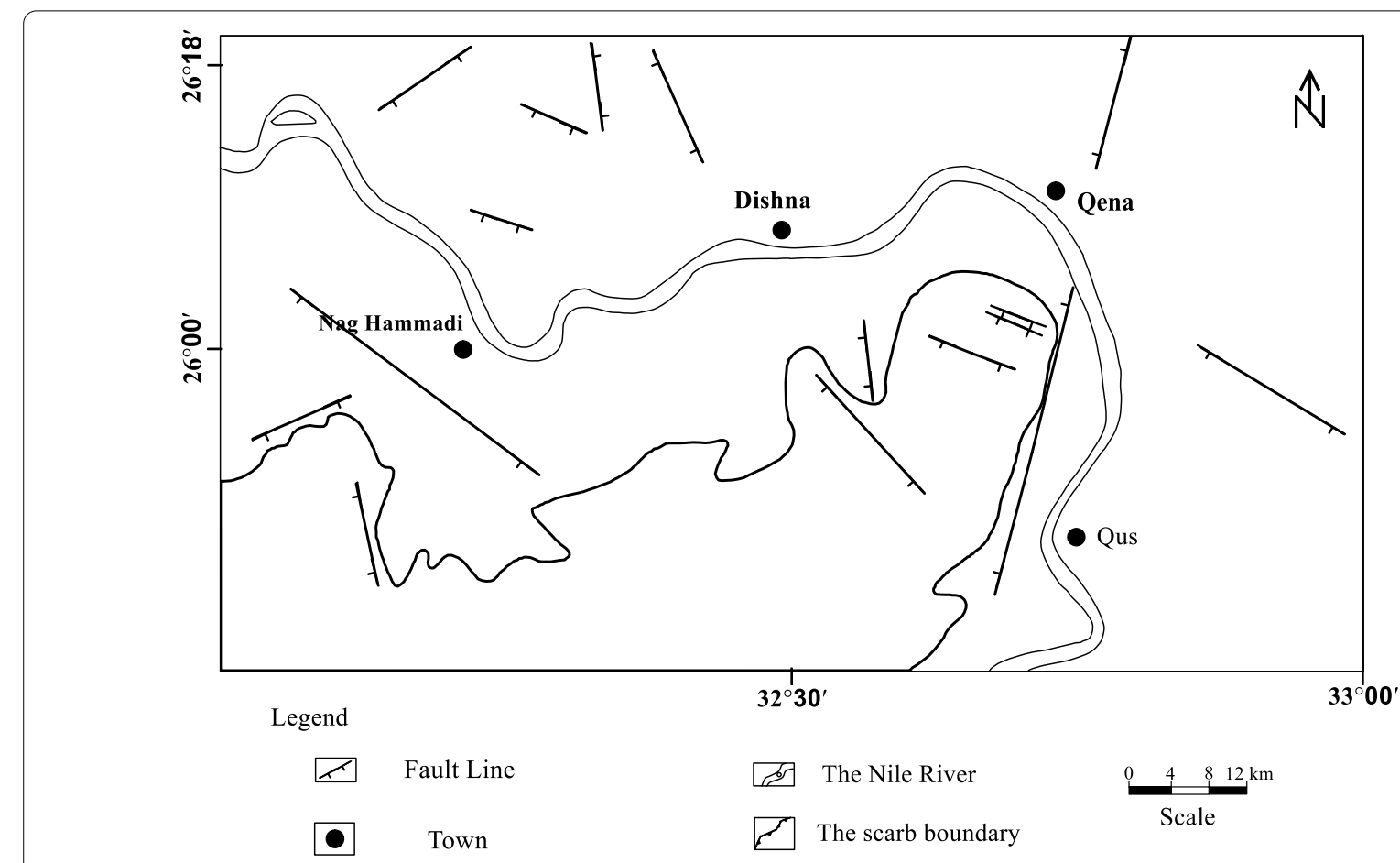

Fig. 4 Structural lineation map of the desert environs west Qena (Said 1962; Youssef 1968) 


\section{Hydrogeological aspects}

The characteristics of the study Quaternary aquifer in both the vertical and horizontal directions are studied through the hydrological cross section in NE-SW direction (Fig. 5) (modified after El Sabri 2010). In this section, the author used new shallow and deep wells drilled in the investigated area and situated at different localities in the cultivated and the desert fringes, in addition to some subsurface geological and geophysical studies collected from (Abd El-Latif et al. 2012; El-Sheikh et al. 2015).

The water in this aquifer is found under semi-confined conditions (under the old cultivated area) and unconfined conditions (under the reclaimed area) where the overlay Nile silts layer is absent. The thickness of the groundwater-bearing layer is different from one location to another within the aquifer and it is about $170 \mathrm{~m}$ recorded thickness.

The hydraulic conductivity of the aquifer which is determined from pumping test ranges from 17.63 to $42.86 \mathrm{~m} /$ day, with an average value of $27.04 \mathrm{~m} /$ day. While the transmissivity ranges between 2142.8 and $1128.3 \mathrm{~m}^{2} /$ day, with an average value of $1456.74 \mathrm{~m}^{2} /$ day. The total depth of the wells in the study area varies from $9 \mathrm{~m}$ in the cultivated land to $215 \mathrm{~m}$ in the newly reclaimed area and

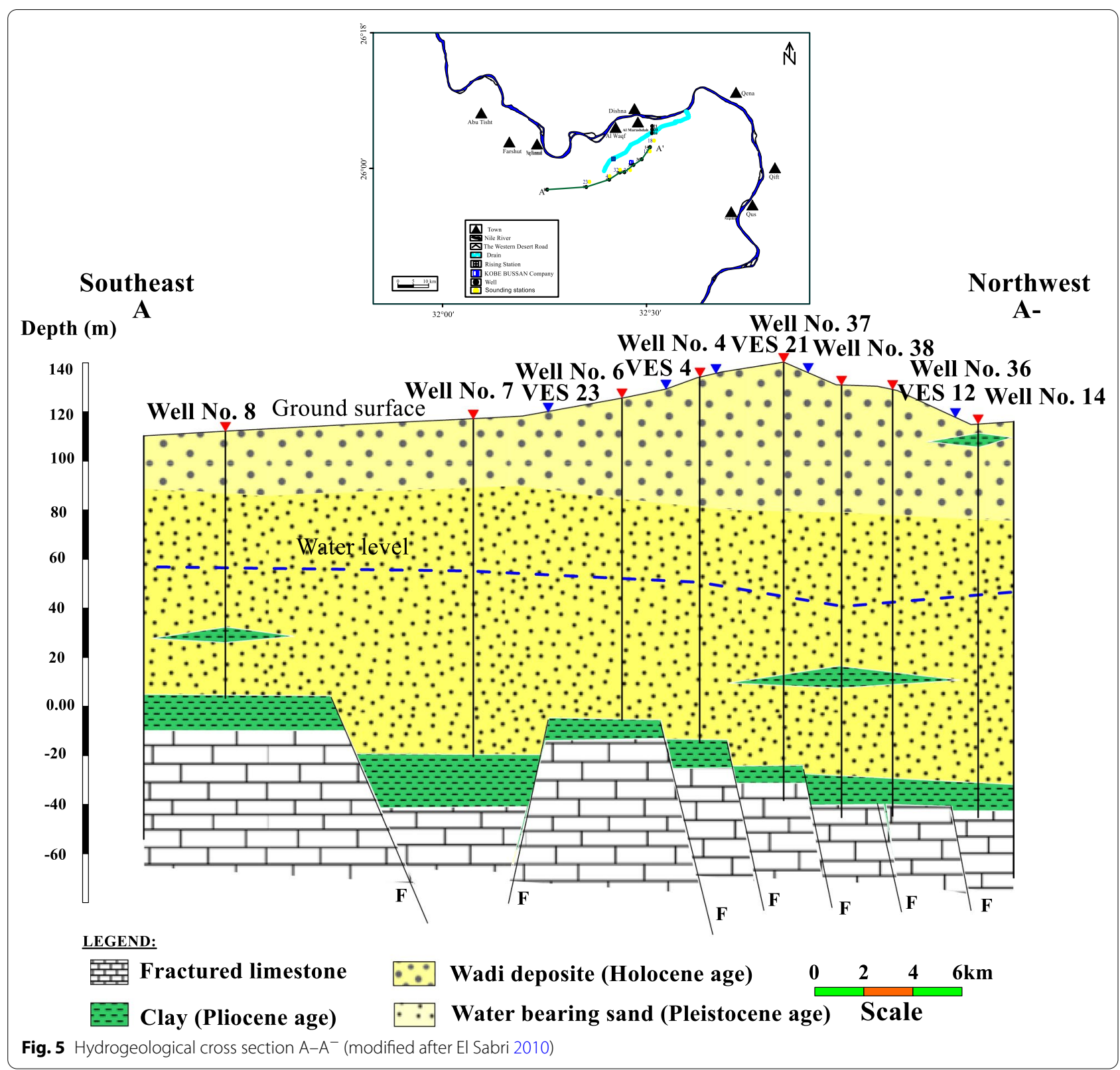


the depth to water ranges between 4 and $97 \mathrm{~m}$. The constructed water level contour map during the year 2016 indicated that the water level decreases regionally from south to north direction and its direction is mainly from southeast to northwest direction, with some local flow groundwater directions from Nile River at the north to old alluvial plain and desert environs at south due to the presence of cone of depression near AL Marashdah and AL Waqf area (Fig. 6 and Table 1).

The Quaternary aquifer is mainly recharged by surface water systems (Nile River, main drainage and irrigation water) while the discharge is mainly represented by Nile River (in some localities), irrigation canals and groundwater wells.

\section{Hydrogeochemical and isotopic characteristics}

The water samples analyses are discussed and interpreted referring to the following items: salinity, water type, hypothetical salt combinations and Piper diagram. This discussion has led to the following results:

1. According to the groundwater salinity distribution map (Fig. 7 and Table 2), the lower values were observed for water samples of the western, central and northeastern parts of the study area (near the desert fringes), while the higher values were observed for water samples of northern and northwestern parts (near Al Marashdah, Al Waqf area and Abu Tisht) and southeastern part (Naqadah area) along the young alluvial valley of the Nile River. The total dissolved solids (TDS) ranged from 589 to $3832 \mathrm{mg} / \mathrm{L}$.

2. According to the concentrations of cations and anions (epm) in the groundwater samples of the Quaternary aquifer, two water chemical types are prevailing in the study area for the Quaternary groundwater samples, indicating the effect of dissolution and ion exchange processes for sediments rich in clay or shale interbeds during the slow movement of groundwater and one water chemical type for Nile River as follows:

\section{i. Chloride-Sodium}

This is the dominant water type (86\%) and characterizes the high salinity groundwater in the study area.

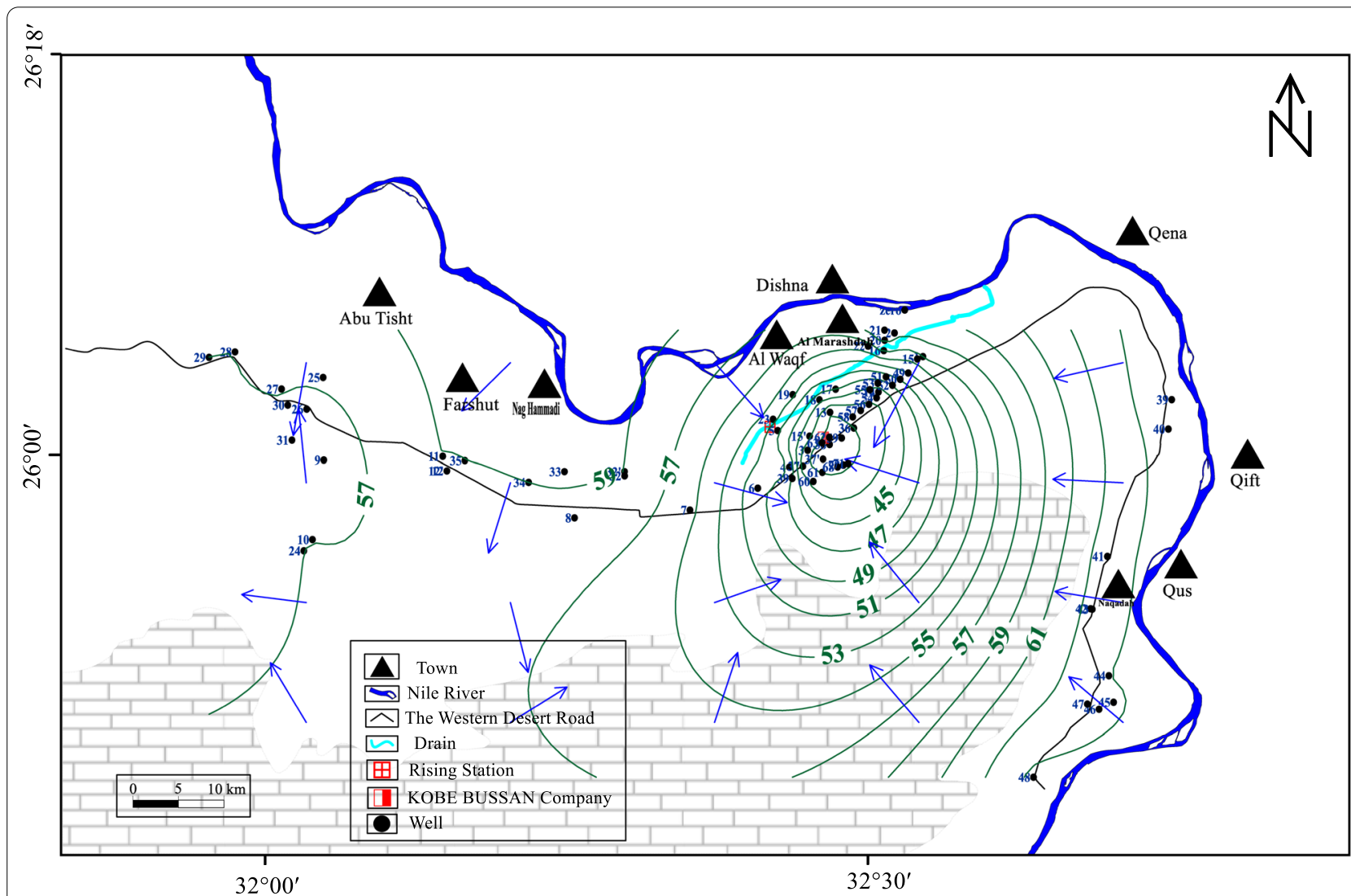

Fig. 6 Water level contour map and flow direction 
Table 1 Hydrogeological data of the selected water points during (Dec. 2016)

\begin{tabular}{|c|c|c|c|c|c|}
\hline Well no & $\begin{array}{l}\text { Depth to water DTW } \\
\text { (m) }\end{array}$ & Total depth T.D. (m) & $\begin{array}{l}\text { Ground elevation G.E. } \\
\text { (m) }\end{array}$ & $\begin{array}{l}\text { Absolute water level } \\
\text { W.L. (m) }\end{array}$ & Aquifer type \\
\hline 1 & 54.00 & 104.00 & 110.00 & 55.00 & Unconfined \\
\hline 2 & 28.00 & 60.00 & 85.00 & 57.00 & Semi-confined \\
\hline 3 & 87.00 & 125.00 & 128.00 & 41.00 & Unconfined \\
\hline 4 & 85.00 & $150-160$ & 134.00 & 49.00 & Unconfined \\
\hline 5 & 61.00 & - & 110.00 & 49.00 & Unconfined \\
\hline 6 & 71.00 & 120.00 & 120.00 & 49.00 & Unconfined \\
\hline 7 & 57.00 & 140.00 & 112.00 & 55.00 & Unconfined \\
\hline 8 & 52.00 & 80.00 & 110.00 & 58.00 & Unconfined \\
\hline 9 & 44.00 & 156.00 & 100.00 & 56.00 & Unconfined \\
\hline 10 & 69.88 & 185.00 & 127.00 & 57.12 & Unconfined \\
\hline 11 & 26.00 & 75.00 & 85.00 & 59.00 & Unconfined \\
\hline 12 & 32.00 & $55-60$ & 90.00 & 58.00 & Unconfined \\
\hline $12^{\prime}$ & 34.00 & $55-60$ & 92.00 & 58.00 & Unconfined \\
\hline 13 & 69.00 & 135.00 & 110.00 & 41.00 & Unconfined \\
\hline 14 & 67.00 & 150.00 & 110.00 & 43.00 & Unconfined \\
\hline 15 & 55.00 & 150.00 & 108.00 & 53.00 & Unconfined \\
\hline $15^{\prime}$ & 61.95 & 150.00 & 105.00 & 43.05 & Unconfined \\
\hline 16 & 35.00 & 56.00 & 90.00 & 55.00 & Unconfined \\
\hline 17 & 43.00 & - & 90.00 & 47.00 & Unconfined \\
\hline 18 & 55.00 & 68.00 & 100.00 & 45.00 & Unconfined \\
\hline 19 & 39.00 & 133.00 & 90.00 & 51.00 & Unconfined \\
\hline 20 & 28.00 & 54.00 & 85.00 & 57.00 & Semi-confined \\
\hline 21 & 23.00 & 86.00 & 82.00 & 59.00 & Semi-confined \\
\hline 22 & 30.00 & 67.00 & 84.00 & 54.00 & Semi-confined \\
\hline 23 & 48.00 & 100.00 & 100.00 & 52.00 & Unconfined \\
\hline 24 & 79.00 & 162.00 & 136.00 & 57.00 & Unconfined \\
\hline 25 & 23.00 & 58.00 & 80.00 & 57.00 & Semi-confined \\
\hline 26 & 45.00 & 136.00 & 101.00 & 56.00 & Unconfined \\
\hline 27 & 33.00 & 120.00 & 90.00 & 57.00 & Unconfined \\
\hline 28 & 60.00 & 130.00 & 117.00 & 57.00 & Unconfined \\
\hline 29 & 75.00 & 400.00 & 132.00 & 57.00 & Unconfined \\
\hline 30 & 43.00 & 100.00 & 99.00 & 56.00 & Unconfined \\
\hline 31 & 48.00 & 90.00 & 104.00 & 56.00 & Unconfined \\
\hline 32 & 32.00 & 130.00 & 91.00 & 59.00 & Unconfined \\
\hline $32^{\prime}$ & 33.00 & 90.00 & 92.00 & 59.00 & Unconfined \\
\hline 33 & 22.00 & - & 82.00 & 60.00 & Unconfined \\
\hline 34 & 28.00 & 65.00 & 87.00 & 59.00 & Unconfined \\
\hline 35 & 17.00 & 13.00 & 76.00 & 59.00 & Unconfined \\
\hline 36 & 90.00 & $150-200$ & 131.00 & 41.00 & Unconfined \\
\hline 37 & 97.00 & $150-200$ & 141.00 & 43.00 & Unconfined \\
\hline 38 & 92.00 & $150-200$ & 132.00 & 40.00 & Unconfined \\
\hline 39 & 8.00 & - & 75.00 & 67.00 & Unconfined \\
\hline 40 & 4.00 & 910 & 70.00 & 66.00 & Unconfined \\
\hline 41 & 21.00 & 33.00 & 85.00 & 64.00 & Semi-confined \\
\hline 42 & 35.00 & 50.00 & 98.00 & 63.00 & Unconfined \\
\hline 43 & 33.00 & 50.00 & 97.00 & 64.00 & Unconfined \\
\hline 44 & 19.00 & 20.00 & 84.00 & 65.00 & Unconfined \\
\hline 45 & 20.50 & $120-130$ & 85.00 & 64.50 & Semi-confined \\
\hline 46 & 27.50 & $42-45$ & 92.00 & 64.50 & Unconfined \\
\hline
\end{tabular}


Table 1 (continued)

\begin{tabular}{llllll}
\hline Well no & $\begin{array}{l}\text { Depth to water DTW } \\
(\mathbf{m})\end{array}$ & Total depth T.D. $(\mathbf{m})$ & $\begin{array}{l}\text { Ground elevation G.E. } \\
(\mathbf{m})\end{array}$ & $\begin{array}{l}\text { Absolute water level } \\
\text { W.L. }(\mathbf{m})\end{array}$ & $\begin{array}{l}\text { Aquifer type } \\
\text { (n) }\end{array}$ \\
\hline 47 & 36.00 & 70.00 & 100.00 & 64.00 & Unconfined \\
48 & 35.50 & 63.00 & 101.00 & 65.50 & Unconfined \\
\hline
\end{tabular}

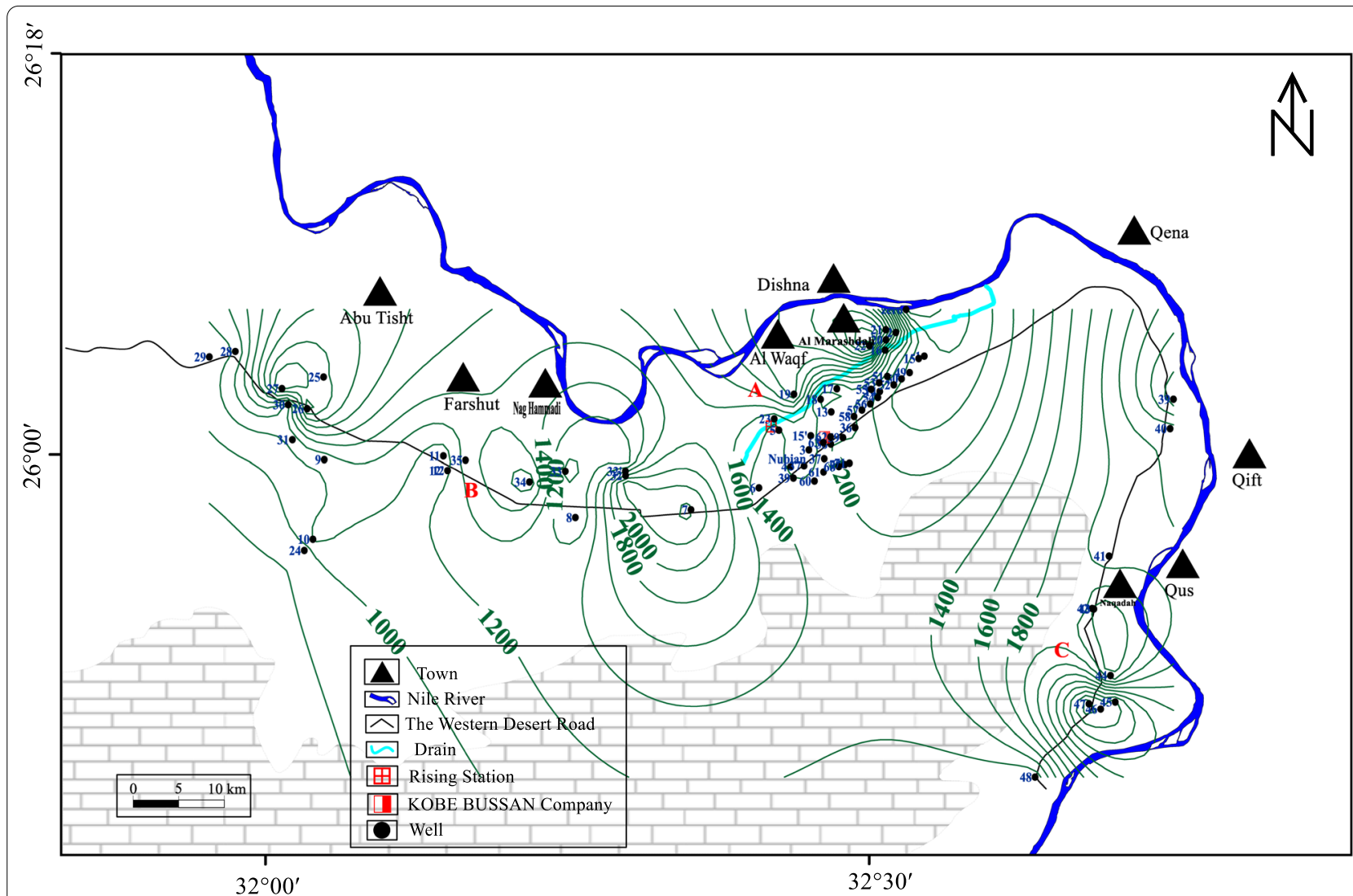

Fig. 7 Iso salinity contour map of groundwater in the desert environs west Qena during (Dec. 2016)

ii. Sulfate-Sodium

This water type rich in sulfate characterizes most of the brackish water wells (14\%).

iii. Bicarbonate-Calcium

It characterizes the fresh Nile River water sample.

3. The detection of four major hypothetical salt groups in groundwater:

\begin{tabular}{|c|c|}
\hline Group-I & $\mathrm{NaCl}, \mathrm{Na}_{2} \mathrm{SO}_{4}, \mathrm{MgSO}_{4}, \mathrm{CaSO}_{4}$, and $\mathrm{Ca}\left(\mathrm{HCO}_{3}\right)_{2}$ \\
\hline Group-II & $\mathrm{NaCl}, \mathrm{MgCl}_{2}, \mathrm{MgSO}_{4}, \mathrm{CaSO}_{4}$, and $\mathrm{Ca}\left(\mathrm{HCO}_{3}\right)_{2}$ \\
\hline Group-III & $\mathrm{NaCl}, \mathrm{Na}_{2} \mathrm{SO}_{4}, \mathrm{MgSO}_{4}, \mathrm{Mg}\left(\mathrm{HCO}_{3}\right)_{2}$, and $\mathrm{Ca}\left(\mathrm{HCO}_{3}\right)_{2}$ \\
\hline Group-IV & $\mathrm{NaCl}, \mathrm{Na}_{2} \mathrm{SO}_{4}, \mathrm{NaHCO}_{3}, \mathrm{Mg}\left(\mathrm{HCO}_{3}\right)_{2}$ and $\mathrm{Ca}\left(\mathrm{HCO}_{3}\right)_{2}$ \\
\hline
\end{tabular}

This is considered as a good indication for both surface water contamination and possible changeable environment of deposition and also reflects the meteoric fresh water origin most probably from the Nile River.

4. The plotting of water samples in Piper diagram (1944) (Fig. 8) indicates that most of water compositions (92\%) are plotted in subarea 7, reflecting primary salinity properties (sodium chloride type), while three groundwater samples are plotted in subarea 9 (fresh water-mixed type) and one sample located in sub-area 6 (calcium chloride type). The major water type in the area is alkaline water with prevailing sulfate and chloride. 
Table 2 Hydrogeochemical data of the selected water samples during (Dec. 2016)

\begin{tabular}{|c|c|c|c|c|c|c|c|c|c|c|c|c|}
\hline \multirow[t]{2}{*}{ Sample no } & \multirow[t]{2}{*}{ EC) Mhos/cm } & \multirow[t]{2}{*}{$\mathrm{PH}$} & \multirow[t]{2}{*}{ TDS (mg/l) } & \multirow[t]{2}{*}{ Units } & \multicolumn{4}{|c|}{ Cations } & \multicolumn{4}{|l|}{ Anions } \\
\hline & & & & & $\mathrm{Ca}^{++}$ & $\mathbf{M g}^{++}$ & $\mathrm{Na}^{+}$ & $\mathbf{K}^{+}$ & $\mathrm{CO}_{3}{ }^{--}$ & $\mathrm{HCO}_{3}{ }^{-}$ & $\mathrm{SO}_{4}^{--}$ & $\mathrm{Cl}^{-}$ \\
\hline \multirow[t]{3}{*}{1} & 1130 & 7.7 & 726 & mg/l & 37.3 & 25.4 & 166.4 & 3.9 & 6 & 122 & 80.5 & 284.8 \\
\hline & & & & meq/l & 1.86 & 2.09 & 7.24 & 0.10 & 0.20 & 2.00 & 1.68 & 8.03 \\
\hline & & & & $\mathrm{e} \%$ & 16.49 & 18.50 & 64.12 & 0.88 & 1.68 & 16.79 & 14.08 & 67.45 \\
\hline \multirow[t]{3}{*}{2} & 2910 & 7.56 & 2143 & mg/l & 86.9 & 48.3 & 522.5 & 3.6 & 18 & 170.8 & 1080.4 & 212.2 \\
\hline & & & & meq/l & 4.34 & 3.97 & 22.73 & 0.09 & 0.60 & 2.80 & 22.49 & 5.98 \\
\hline & & & & $\mathrm{e} \%$ & 13.93 & 12.76 & 73.01 & 0.30 & 1.88 & 8.78 & 70.56 & 18.77 \\
\hline \multirow[t]{3}{*}{3} & 2500 & 7.5 & 1528 & mg/l & 115.3 & 45.3 & 329 & 6.4 & 0 & 97.6 & 263.1 & 671.6 \\
\hline & & & & meq/l & 5.75 & 3.73 & 14.31 & 0.16 & 0.00 & 1.60 & 5.48 & 18.94 \\
\hline & & & & $\mathrm{e} \%$ & 24.02 & 15.55 & 59.75 & 0.68 & 0.00 & 6.15 & 21.05 & 72.80 \\
\hline \multirow[t]{3}{*}{4} & 2220 & 7.52 & 1431 & mg/l & 71 & 46.3 & 345.1 & 6.6 & 9 & 222.7 & 202.5 & 527.4 \\
\hline & & & & meq/l & 3.54 & 3.81 & 15.01 & 0.17 & 0.30 & 3.65 & 4.22 & 14.87 \\
\hline & & & & $\mathrm{e} \%$ & 15.72 & 16.90 & 66.63 & 0.75 & 1.30 & 15.84 & 18.30 & 64.56 \\
\hline \multirow[t]{3}{*}{5} & 2200 & 7.79 & 1355 & mg/l & 68.2 & 32.9 & 366.2 & 5.7 & 12 & 115.9 & 163.8 & 590 \\
\hline & & & & meq/l & 3.40 & 2.71 & 15.93 & 0.15 & 0.40 & 1.90 & 3.41 & 16.64 \\
\hline & & & & $\mathrm{e} \%$ & 15.34 & 12.20 & 71.81 & 0.66 & 1.79 & 8.50 & 15.26 & 74.45 \\
\hline \multirow[t]{3}{*}{6} & 2030 & 7.8 & 1266 & mg/l & 121.1 & 65.6 & 236 & 5.3 & 18 & 134.2 & 146.7 & 538.8 \\
\hline & & & & meq/l & 6.04 & 5.39 & 10.27 & 0.14 & 0.60 & 2.20 & 3.05 & 15.19 \\
\hline & & & & $\mathrm{e} \%$ & 27.67 & 24.70 & 47.01 & 0.62 & 2.85 & 10.45 & 14.51 & 72.19 \\
\hline \multirow[t]{3}{*}{7} & 4040 & 7.63 & 2477 & mg/l & 203.1 & 87.1 & 558 & 8.7 & 9 & 67.1 & 436 & 1108.2 \\
\hline & & & & meq/l & 10.13 & 7.16 & 24.27 & 0.22 & 0.30 & 1.10 & 9.08 & 31.25 \\
\hline & & & & $\mathrm{e} \%$ & 24.25 & 17.14 & 58.08 & 0.53 & 0.72 & 2.64 & 21.75 & 74.89 \\
\hline \multirow[t]{3}{*}{8} & 1540 & 8.18 & 950 & $\mathrm{mg} / \mathrm{l}$ & 21.8 & 11 & 285.3 & 3.9 & 12 & 109.8 & 114.8 & 391.2 \\
\hline & & & & meq/l & 1.09 & 0.90 & 12.41 & 0.10 & 0.40 & 1.80 & 2.39 & 11.03 \\
\hline & & & & $\mathrm{e} \%$ & 7.50 & 6.24 & 85.57 & 0.69 & 2.56 & 11.52 & 15.30 & 70.62 \\
\hline \multirow[t]{3}{*}{9} & 2130 & 7.77 & 1262 & mg/l & 113.9 & 51.5 & 270.7 & 6 & 24 & 67.1 & 156.4 & 572.2 \\
\hline & & & & meq/l & 5.68 & 4.24 & 11.78 & 0.15 & 0.80 & 1.10 & 3.26 & 16.14 \\
\hline & & & & $\mathrm{e} \%$ & 26.01 & 19.39 & 53.90 & 0.70 & 3.76 & 5.17 & 15.29 & 75.78 \\
\hline \multirow[t]{3}{*}{10} & 2060 & 8.06 & 1263 & mg/l & 37.6 & 23.2 & 368.5 & 3.5 & 0 & 134.2 & 149 & 547 \\
\hline & & & & meq/l & 1.88 & 1.91 & 16.03 & 0.09 & 0.00 & 2.20 & 3.10 & 15.43 \\
\hline & & & & $\mathrm{e} \%$ & 9.43 & 9.59 & 80.54 & 0.45 & 0.00 & 10.61 & 14.97 & 74.42 \\
\hline \multirow[t]{3}{*}{11} & 1630 & 7.97 & 1055 & $\mathrm{mg} / \mathrm{l}$ & 56.8 & 30 & 256.1 & 2.9 & 18 & 195.2 & 110.6 & 385.9 \\
\hline & & & & meq/l & 2.83 & 2.47 & 11.14 & 0.07 & 0.60 & 3.20 & 2.30 & 10.88 \\
\hline & & & & $\mathrm{e} \%$ & 17.16 & 14.94 & 67.45 & 0.45 & 3.53 & 18.84 & 13.56 & 64.07 \\
\hline 12 & 1780 & 8.08 & 1150 & mg/l & 52.6 & 31.7 & 299.9 & 4.6 & 12 & 176.9 & 126 & 446.8 \\
\hline & & & & meq/l & 2.62 & 2.61 & 13.05 & 0.12 & 0.40 & 2.90 & 2.62 & 12.60 \\
\hline & & & & $\mathrm{e} \%$ & 14.27 & 14.17 & 70.92 & 0.64 & 2.16 & 15.65 & 14.16 & 68.02 \\
\hline $12^{\prime}$ & 2770 & 8.05 & 1901 & $\mathrm{mg} / \mathrm{l}$ & 61.8 & 33.7 & 518.4 & 5.8 & 9 & 176.9 & 700.4 & 394.7 \\
\hline & & & & meq/l & 3.08 & 2.77 & 22.55 & 0.15 & 0.30 & 2.90 & 14.58 & 11.13 \\
\hline & & & & e\% & 10.80 & 9.71 & 78.97 & 0.52 & 1.04 & 10.03 & 50.44 & 38.50 \\
\hline 13 & 2320 & 8.06 & 1448 & mg/l & 58.1 & 37.7 & 389.6 & 12.1 & 12 & 158.6 & 150.2 & 629.6 \\
\hline & & & & meq/l & 2.90 & 3.10 & 16.95 & 0.31 & 0.40 & 2.60 & 3.13 & 17.75 \\
\hline & & & & $\mathrm{e} \%$ & 12.47 & 13.33 & 72.87 & 1.33 & 1.67 & 10.88 & 13.09 & 74.35 \\
\hline 14 & 1570 & 8.23 & 907 & mg/l & 34 & 27.6 & 229.4 & 7 & 6 & 97.6 & 138 & 367.8 \\
\hline & & & & meq/l & 1.70 & 2.27 & 9.98 & 0.18 & 0.20 & 1.60 & 2.87 & 10.37 \\
\hline & & & & $\mathrm{e} \%$ & 12.01 & 16.07 & 70.65 & 1.27 & 1.33 & 10.63 & 19.10 & 68.94 \\
\hline 15 & 950 & 8.23 & 589 & meq/l & 1.39 & 1.67 & 6.07 & 0.10 & 0.20 & 2.35 & 1.41 & 5.07 \\
\hline & & & & e\% & 15.08 & 18.09 & 65.75 & 1.08 & 2.21 & 26.01 & 15.64 & 56.14 \\
\hline & & & & $\mathrm{mg} / \mathrm{l}$ & 94.7 & 48.1 & 340.5 & 7.8 & 6 & 149.5 & 507.8 & 395 \\
\hline
\end{tabular}


Table 2 (continued)

\begin{tabular}{|c|c|c|c|c|c|c|c|c|c|c|c|c|}
\hline \multirow[t]{2}{*}{ Sample no } & \multirow[t]{2}{*}{ EC) Mhos/cm } & \multirow[t]{2}{*}{$\mathrm{PH}$} & \multirow[t]{2}{*}{ TDS (mg/l) } & \multirow[t]{2}{*}{ Units } & \multicolumn{4}{|c|}{ Cations } & \multicolumn{4}{|l|}{ Anions } \\
\hline & & & & & $\mathrm{Ca}^{++}$ & $\mathbf{M g}^{++}$ & $\mathrm{Na}^{+}$ & $\mathbf{K}^{+}$ & $\mathrm{CO}_{3}^{--}$ & $\mathrm{HCO}_{3}{ }^{-}$ & $\mathrm{SO}_{4}^{--}$ & $\mathrm{Cl}^{-}$ \\
\hline \multirow[t]{3}{*}{16} & 2470 & 8.15 & 1549 & meq/l & 4.73 & 3.96 & 14.81 & 0.20 & 0.20 & 2.45 & 10.57 & 11.14 \\
\hline & & & & $\mathrm{e} \%$ & 19.95 & 16.70 & 62.52 & 0.84 & 0.82 & 10.06 & 43.40 & 45.72 \\
\hline & & & & $\mathrm{mg} / \mathrm{l}$ & 69.2 & 53.6 & 264.3 & 5.6 & 18 & 122 & 220.4 & 417.2 \\
\hline \multirow[t]{3}{*}{17} & 2080 & 8.05 & 1170 & meq/l & 3.45 & 4.41 & 11.50 & 0.14 & 0.60 & 2.00 & 4.59 & 11.77 \\
\hline & & & & $\mathrm{e} \%$ & 17.71 & 22.60 & 58.95 & 0.73 & 3.17 & 10.55 & 24.21 & 62.07 \\
\hline & & & & mg/l & 34.5 & 31.9 & 235.5 & 4.7 & 6 & 131.2 & 106.5 & 390.6 \\
\hline \multirow[t]{3}{*}{18} & 1530 & 8.25 & 941 & meq/l & 1.72 & 2.62 & 10.24 & 0.12 & 0.20 & 2.15 & 2.22 & 11.01 \\
\hline & & & & $\mathrm{e} \%$ & 11.70 & 17.84 & 69.64 & 0.82 & 1.28 & 13.80 & 14.23 & 70.69 \\
\hline & & & & mg/l & 179.6 & 106.9 & 533.8 & 9.6 & 6 & 125.1 & 696 & 934.2 \\
\hline \multirow[t]{3}{*}{19} & 4050 & 7.94 & 2591 & meq/l & 8.96 & 8.79 & 23.22 & 0.25 & 0.20 & 2.05 & 14.49 & 26.34 \\
\hline & & & & $\mathrm{e} \%$ & 21.74 & 21.33 & 56.33 & 0.60 & 0.46 & 4.76 & 33.63 & 61.14 \\
\hline & & & & $\mathrm{mg} / \mathrm{l}$ & 153.3 & 77.5 & 541.9 & 7.5 & 18 & 115.9 & 1190 & 378 \\
\hline \multirow[t]{3}{*}{20} & 3530 & 8.13 & 2482 & meq/l & 7.65 & 6.37 & 23.57 & 0.19 & 0.60 & 1.90 & 24.78 & 10.66 \\
\hline & & & & $\mathrm{e} \%$ & 20.24 & 16.87 & 62.38 & 0.51 & 1.58 & 5.01 & 65.31 & 28.10 \\
\hline & & & & mg/l & 264.2 & 122.4 & 522.9 & 12.9 & 18 & 176.9 & 1225.9 & 590.8 \\
\hline \multirow[t]{3}{*}{21} & 4310 & 7.93 & 2934 & meq/l & 13.18 & 10.07 & 22.75 & 0.33 & 0.60 & 2.90 & 25.52 & 16.66 \\
\hline & & & & $\mathrm{e} \%$ & 28.46 & 21.73 & 49.10 & 0.71 & 1.31 & 6.35 & 55.87 & 36.47 \\
\hline & & & & mg/l & 296.9 & 141.6 & 768.9 & 11.5 & 12 & 134.2 & 1124.3 & 1342.5 \\
\hline \multirow[t]{3}{*}{22} & 6220 & 7.84 & 3832 & meq/l & 14.82 & 11.65 & 33.45 & 0.29 & 0.40 & 2.20 & 23.41 & 37.86 \\
\hline & & & & $\mathrm{e} \%$ & 24.61 & 19.34 & 55.56 & 0.49 & 0.63 & 3.44 & 36.65 & 59.28 \\
\hline & & & & $\mathrm{mg} / \mathrm{l}$ & 120.6 & 49.7 & 289.3 & 4.6 & 24 & 85.4 & 275.7 & 571.5 \\
\hline \multirow[t]{3}{*}{23} & 2350 & 8.06 & 1421 & meq/l & 6.02 & 4.09 & 12.58 & 0.12 & 0.80 & 1.40 & 5.74 & 16.12 \\
\hline & & & & $\mathrm{e} \%$ & 26.39 & 17.92 & 55.18 & 0.52 & 3.33 & 5.82 & 23.86 & 66.99 \\
\hline & & & & $\mathrm{mg} / \mathrm{l}$ & 15.7 & 4.9 & 289.3 & 2.5 & 12 & 115.9 & 105.2 & 318.3 \\
\hline \multirow[t]{3}{*}{24} & 1380 & 8.57 & 864 & meq/l & 0.78 & 0.40 & 12.58 & 0.06 & 0.40 & 1.90 & 2.19 & 8.98 \\
\hline & & & & $\mathrm{e} \%$ & 5.66 & 2.91 & 90.96 & 0.46 & 2.97 & 14.11 & 16.27 & 66.66 \\
\hline & & & & $\mathrm{mg} / \mathrm{l}$ & 179.3 & 82.9 & 518.2 & 10.4 & 12 & 176.9 & 473.8 & 976.9 \\
\hline \multirow[t]{3}{*}{25} & 4120 & 7.75 & 2430 & meq/l & 8.95 & 6.82 & 22.54 & 0.27 & 0.40 & 2.90 & 9.86 & 27.55 \\
\hline & & & & $\mathrm{e} \%$ & 23.20 & 17.68 & 58.44 & 0.69 & 0.98 & 7.12 & 24.23 & 67.67 \\
\hline & & & & mg/l & 325.1 & 138.3 & 405.8 & 10.4 & 6 & 103.7 & 470.1 & 1257.5 \\
\hline \multirow[t]{3}{*}{26} & 4380 & 7.9 & 717 & meq/l & 16.22 & 11.37 & 17.65 & 0.27 & 0.20 & 1.70 & 9.79 & 35.46 \\
\hline & & & & $\mathrm{e} \%$ & 35.64 & 24.99 & 38.78 & 0.58 & 0.42 & 3.60 & 20.76 & 75.21 \\
\hline & & & & $\mathrm{e} \%$ & 35.64 & 24.99 & 38.78 & 0.58 & 0.42 & 3.60 & 20.76 & 75.21 \\
\hline 27 & 4690 & 8.08 & 2939 & mg/l & 188.5 & 84 & 690.4 & 8.8 & 12 & 274.5 & 647.9 & 1032.5 \\
\hline & & & & meq/l & 9.41 & 6.91 & 30.03 & 0.23 & 0.40 & 4.50 & 13.49 & 29.12 \\
\hline & & & & $\mathrm{e} \%$ & 20.20 & 14.83 & 64.49 & 0.48 & 0.84 & 9.47 & 28.40 & 61.29 \\
\hline 28 & 1990 & 8.21 & 1190 & mg/l & 51.9 & 41.8 & 281.4 & 5.9 & 9 & 128.1 & 210.2 & 462.2 \\
\hline & & & & meq/l & 2.59 & 3.44 & 12.24 & 0.15 & 0.30 & 2.10 & 4.38 & 13.03 \\
\hline & & & & e\% & 14.06 & 18.66 & 66.46 & 0.82 & 1.51 & 10.60 & 22.09 & 65.80 \\
\hline 29 & 1810 & 8.33 & 1101 & mg/l & 38.7 & 12.7 & 320 & 7 & 6 & 149.5 & 71.8 & 495 \\
\hline & & & & meq/l & 1.93 & 1.04 & 13.92 & 0.18 & 0.20 & 2.45 & 1.49 & 13.96 \\
\hline & & & & $\mathrm{e} \%$ & 11.28 & 6.10 & 81.57 & 1.05 & 1.10 & 13.53 & 8.26 & 77.10 \\
\hline 30 & 2060 & 8.17 & 1238 & mg/l & 96 & 49.6 & 250 & 4.1 & 12 & 140.3 & 189.2 & 496.8 \\
\hline & & & & meq/l & 4.79 & 4.08 & 10.88 & 0.10 & 0.40 & 2.30 & 3.94 & 14.01 \\
\hline & & & & $\mathrm{e} \%$ & 24.13 & 20.55 & 54.79 & 0.53 & 1.94 & 11.14 & 19.08 & 67.85 \\
\hline 31 & 2110 & 8.14 & 1275 & mg/l & 101.8 & 32.6 & 276.9 & 5.5 & 6 & 140.3 & 147.1 & 565.2 \\
\hline & & & & meq/l & 5.08 & 2.68 & 12.05 & 0.14 & 0.20 & 2.30 & 3.06 & 15.94 \\
\hline & & & & $\mathrm{e} \%$ & 25.47 & 13.44 & 60.39 & 0.71 & 0.93 & 10.70 & 14.24 & 74.13 \\
\hline
\end{tabular}


Table 2 (continued)

\begin{tabular}{|c|c|c|c|c|c|c|c|c|c|c|c|c|}
\hline \multirow[t]{2}{*}{ Sample no } & \multirow[t]{2}{*}{ EC) Mhos/cm } & \multirow[t]{2}{*}{$\mathrm{PH}$} & \multirow[t]{2}{*}{ TDS (mg/l) } & \multirow[t]{2}{*}{ Units } & \multicolumn{4}{|c|}{ Cations } & \multicolumn{4}{|l|}{ Anions } \\
\hline & & & & & $\mathrm{Ca}^{++}$ & $\mathbf{M g}^{++}$ & $\mathrm{Na}^{+}$ & $\mathbf{K}^{+}$ & $\mathrm{CO}_{3}^{--}$ & $\mathrm{HCO}_{3}{ }^{-}$ & $\mathrm{SO}_{4}^{--}$ & $\mathrm{Cl}^{-}$ \\
\hline \multirow[t]{3}{*}{32} & 3580 & 7.96 & 2420 & mg/l & 175 & 92 & 480.9 & 9.2 & 6 & 140.3 & 805.9 & 710.4 \\
\hline & & & & meq/l & 8.73 & 7.57 & 20.92 & 0.24 & 0.20 & 2.30 & 16.78 & 20.03 \\
\hline & & & & $\mathrm{e} \%$ & 23.32 & 20.20 & 55.85 & 0.63 & 0.51 & 5.85 & 42.68 & 50.96 \\
\hline \multirow[t]{3}{*}{$32^{\prime}$} & 1600 & 7.3 & 1078 & mg/l & 69.5 & 38.9 & 217.5 & 2.1 & 0 & 195.2 & 253.8 & 301.5 \\
\hline & & & & meq/l & 3.47 & 3.20 & 9.46 & 0.05 & 0.00 & 3.20 & 5.28 & 8.50 \\
\hline & & & & $\mathrm{e} \%$ & 21.43 & 19.77 & 58.47 & 0.33 & 0.00 & 18.84 & 31.11 & 50.06 \\
\hline \multirow[t]{3}{*}{33} & 1270 & 8.27 & 892 & mg/l & 79.5 & 37.8 & 137.9 & 4.1 & 6 & 280.6 & 244.6 & 102 \\
\hline & & & & meq/l & 3.97 & 3.11 & 6.00 & 0.10 & 0.20 & 4.60 & 5.09 & 2.88 \\
\hline & & & & $\mathrm{e} \%$ & 30.10 & 23.59 & 45.52 & 0.80 & 1.57 & 36.02 & 39.89 & 22.53 \\
\hline \multirow[t]{3}{*}{34} & 2710 & 8.25 & 1947 & mg/l & 119.9 & 62.1 & 393.7 & 3.3 & 12 & 176.9 & 734.1 & 444.8 \\
\hline & & & & meq/l & 5.98 & 5.11 & 17.13 & 0.08 & 0.40 & 2.90 & 15.28 & 12.54 \\
\hline & & & & e\% & 21.14 & 18.05 & 60.51 & 0.30 & 1.28 & 9.31 & 49.10 & 40.30 \\
\hline \multirow[t]{3}{*}{35} & 2260 & 8.35 & 1526 & mg/l & 49.7 & 30 & 397.5 & 4.8 & 24 & 302 & 353.1 & 364.5 \\
\hline & & & & meq/l & 2.48 & 2.47 & 17.29 & 0.12 & 0.80 & 4.95 & 7.35 & 10.28 \\
\hline & & & & $\mathrm{e} \%$ & 11.08 & 11.10 & 77.27 & 0.55 & 3.42 & 21.17 & 31.44 & 43.96 \\
\hline \multirow[t]{3}{*}{36} & 2060 & 8.43 & 1250 & $\mathrm{mg} / \mathrm{l}$ & 43.7 & 32.5 & 339.8 & 5.1 & 6 & 115.9 & 225.5 & 481.3 \\
\hline & & & & meq/l & 2.18 & 2.67 & 14.78 & 0.13 & 0.20 & 1.90 & 4.69 & 13.57 \\
\hline & & & & $\mathrm{e} \%$ & 11.03 & 13.52 & 74.78 & 0.66 & 0.98 & 9.33 & 23.05 & 66.64 \\
\hline \multirow[t]{3}{*}{37} & 2390 & 8.26 & 1470 & mg/l & 76.4 & 48.5 & 343.7 & 6.8 & 12 & 198.3 & 246.8 & 537.6 \\
\hline & & & & meq/l & 3.81 & 3.99 & 14.95 & 0.17 & 0.40 & 3.25 & 5.14 & 15.16 \\
\hline & & & & $\mathrm{e} \%$ & 16.63 & 17.40 & 65.21 & 0.76 & 1.67 & 13.57 & 21.46 & 63.30 \\
\hline \multirow[t]{3}{*}{38} & 1940 & 8.5 & 1167 & $\mathrm{mg} / \mathrm{l}$ & 53.6 & 22.9 & 320.6 & 5.2 & 12 & 109.8 & 209.6 & 433.8 \\
\hline & & & & meq/l & 2.67 & 1.88 & 13.95 & 0.13 & 0.40 & 1.80 & 4.36 & 12.23 \\
\hline & & & & $\mathrm{e} \%$ & 14.35 & 10.11 & 74.83 & 0.71 & 2.13 & 9.57 & 23.22 & 65.08 \\
\hline \multirow[t]{3}{*}{39} & 4240 & 8.04 & 2962 & mg/l & 120.3 & 69.2 & 723.4 & 15.4 & 9 & 289.8 & 1145.6 & 589.5 \\
\hline & & & & meq/l & 6.00 & 5.69 & 31.47 & 0.39 & 0.30 & 4.75 & 23.85 & 16.62 \\
\hline & & & & $\mathrm{e} \%$ & 13.78 & 13.07 & 72.25 & 0.90 & 0.66 & 10.43 & 52.39 & 36.52 \\
\hline \multirow[t]{3}{*}{40} & 3490 & 8.47 & 2266 & $\mathrm{mg} / \mathrm{l}$ & 49.7 & 45.8 & 625.6 & 13.4 & 24 & 286.7 & 473.4 & 747.9 \\
\hline & & & & meq/l & 2.48 & 3.77 & 27.21 & 0.34 & 0.80 & 4.70 & 9.86 & 21.09 \\
\hline & & & & $\mathrm{e} \%$ & 7.34 & 11.14 & 80.51 & 1.01 & 2.19 & 12.89 & 27.04 & 57.87 \\
\hline \multirow[t]{3}{*}{41} & 2990 & 8.6 & 2130 & mg/l & 26.4 & 18.1 & 685.3 & 12.4 & 12 & 146.4 & 614.6 & 614.4 \\
\hline & & & & meq/l & 1.32 & 1.49 & 29.81 & 0.32 & 0.40 & 2.40 & 12.80 & 17.33 \\
\hline & & & & $\mathrm{e} \%$ & 4.00 & 4.52 & 90.52 & 0.96 & 1.21 & 7.29 & 38.87 & 52.63 \\
\hline 42 & 2630 & 8.42 & 1838 & $\mathrm{mg} / \mathrm{l}$ & 59.1 & 16.8 & 531.3 & 12.7 & 6 & 222.7 & 371.8 & 617.7 \\
\hline & & & & meq/l & 2.95 & 1.38 & 23.11 & 0.32 & 0.20 & 3.65 & 7.74 & 17.42 \\
\hline & & & & e \% & 10.62 & 4.98 & 83.23 & 1.17 & 0.69 & 12.58 & 26.68 & 60.05 \\
\hline 43 & 2030 & 8.62 & 1409 & mg/l & 34.5 & 11.6 & 406 & 11.2 & 18 & 231.8 & 341.6 & 354.4 \\
\hline & & & & meq/l & 1.72 & 0.95 & 17.66 & 0.29 & 0.60 & 3.80 & 7.11 & 9.99 \\
\hline & & & & $\mathrm{e} \%$ & 8.35 & 4.63 & 85.64 & 1.39 & 2.79 & 17.67 & 33.07 & 46.47 \\
\hline 44 & 2300 & 8.26 & 1452 & mg/l & 102.9 & 77.5 & 283.8 & 20.2 & 18 & 207.4 & 309.4 & 432.5 \\
\hline & & & & meq/l & 5.13 & 6.37 & 12.35 & 0.52 & 0.60 & 3.40 & 6.44 & 12.20 \\
\hline & & & & $\mathrm{e} \%$ & 21.07 & 26.15 & 50.66 & 2.12 & 2.65 & 15.02 & 28.46 & 53.88 \\
\hline 45 & 4390 & 8.22 & 3330 & $\mathrm{mg} / \mathrm{l}$ & 174.6 & 126.9 & 753.2 & 27.5 & 0 & 308.1 & 935.9 & 1004.3 \\
\hline & & & & meq/l & 8.71 & 10.44 & 32.76 & 0.70 & 0.00 & 5.05 & 19.49 & 28.32 \\
\hline & & & & $\mathrm{e} \%$ & 16.56 & 19.83 & 62.27 & 1.34 & 0.00 & 9.55 & 36.86 & 53.58 \\
\hline 46 & 4600 & 8.3 & 3256 & mg/l & 126.6 & 133.8 & 771.1 & 19.4 & 0 & 173.9 & 693.2 & 1337.7 \\
\hline & & & & meq/l & 6.32 & 11.00 & 33.54 & 0.50 & 0.00 & 2.85 & 14.43 & 37.72 \\
\hline & & & & e\% & 12.30 & 21.42 & 65.31 & 0.97 & 0.00 & 5.18 & 26.24 & 68.58 \\
\hline
\end{tabular}


Table 2 (continued)

\begin{tabular}{|c|c|c|c|c|c|c|c|c|c|c|c|c|}
\hline \multirow[t]{2}{*}{ Sample no } & \multirow[t]{2}{*}{ EC) Mhos/cm } & \multirow[t]{2}{*}{$\mathrm{PH}$} & \multirow[t]{2}{*}{ TDS (mg/l) } & \multirow[t]{2}{*}{ Units } & \multicolumn{4}{|c|}{ Cations } & \multicolumn{4}{|c|}{ Anions } \\
\hline & & & & & $\mathrm{Ca}^{++}$ & $\mathbf{M g}^{++}$ & $\mathrm{Na}^{+}$ & $\mathbf{K}^{+}$ & $\mathrm{CO}_{3}^{--}$ & $\mathrm{HCO}_{3}^{-}$ & $\mathrm{SO}_{4}^{--}$ & $\mathrm{Cl}^{-}$ \\
\hline \multirow[t]{3}{*}{47} & 5090 & 8.23 & 3684 & mg/l & 164.5 & 133.5 & 888.4 & 23.6 & 6 & 146.4 & 735.2 & 1586.5 \\
\hline & & & & meq/l & 8.21 & 10.98 & 38.65 & 0.60 & 0.20 & 2.40 & 15.31 & 44.74 \\
\hline & & & & $\mathrm{e} \%$ & 14.05 & 18.79 & 66.13 & 1.03 & 0.32 & 3.83 & 24.43 & 71.42 \\
\hline \multirow[t]{3}{*}{48} & 1770 & 8.53 & 1038 & mg/l & 18.3 & 6 & 319.9 & 6.7 & 24 & 192.2 & 133.6 & 337.5 \\
\hline & & & & meq/l & 0.91 & 0.49 & 13.92 & 0.17 & 0.80 & 3.15 & 2.78 & 9.52 \\
\hline & & & & $\mathrm{e} \%$ & 5.89 & 3.18 & 89.82 & 1.11 & 4.92 & 19.39 & 17.12 & 58.57 \\
\hline \multirow[t]{3}{*}{ Zero } & 350 & 8.44 & 228 & mg/l & 27.8 & 10.5 & 21.8 & 4.6 & 6 & 122 & 23.9 & 11.8 \\
\hline & & & & meq/l & 1.39 & 0.86 & 0.95 & 0.12 & 0.20 & 2.00 & 0.50 & 0.33 \\
\hline & & & & $\mathrm{e} \%$ & 41.83 & 26.04 & 28.59 & 3.55 & 6.60 & 65.99 & 16.42 & 10.98 \\
\hline
\end{tabular}

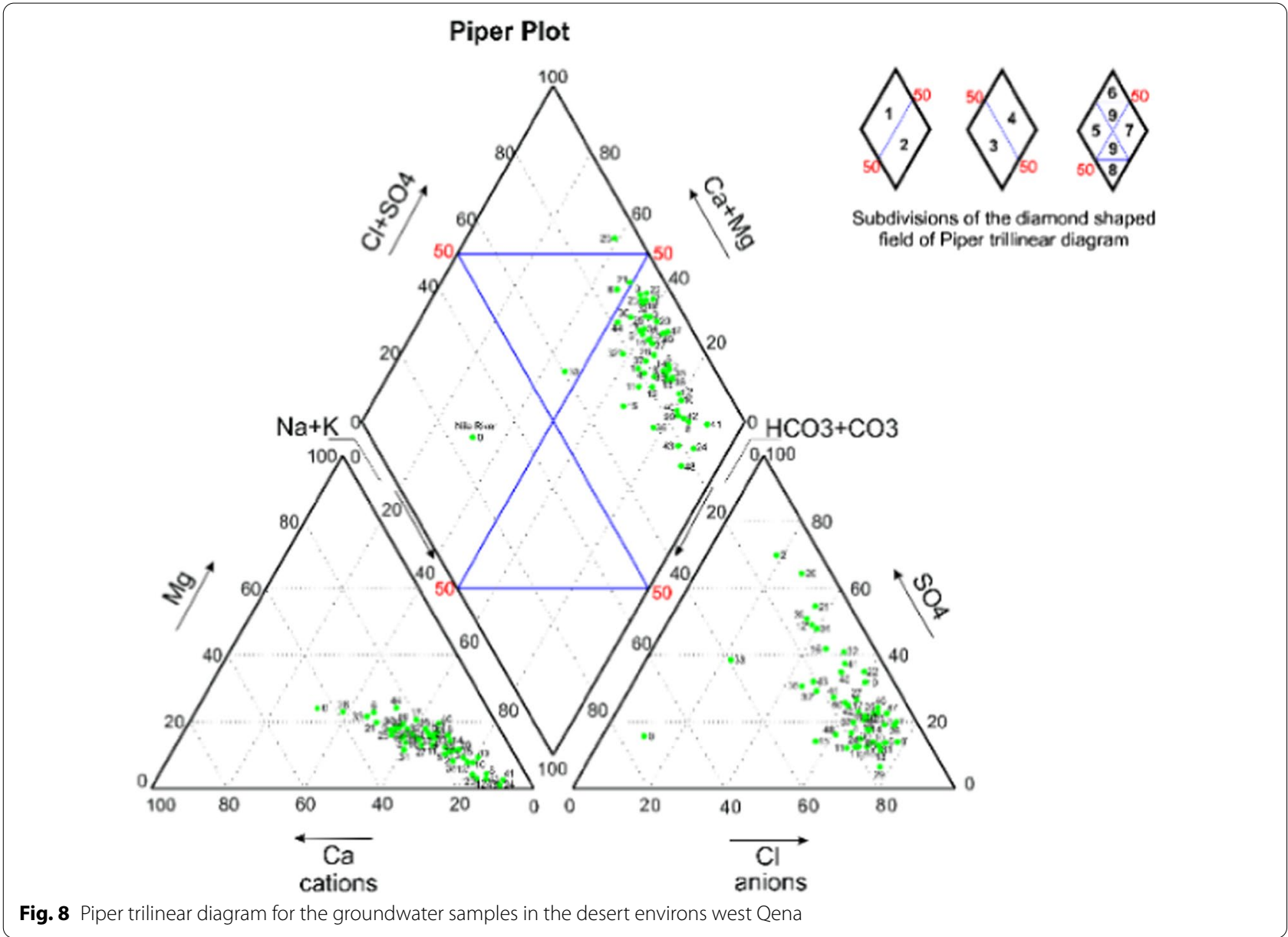

5. Discharging, agricultural, industrial and domestic disposals mostly cause the high concentration of trace elements. The study of the distribution of such elements $\left(\mathrm{SiO}_{2}, \mathrm{~B}^{3+}, \mathrm{Al}^{3+}, \mathrm{Li}^{+}, \mathrm{Mo}, \mathrm{Sr}^{2+}, \mathrm{Ba}^{2+}, \mathrm{Cr}\right.$, $\mathrm{Cu}, \mathrm{Fe}^{2+}, \mathrm{Mn}^{2+}, \mathrm{Ni}, \mathrm{Pb}, \mathrm{V}$ and $\mathrm{Zn}$ ) in groundwater samples (Table 3) indicates that some of the ground- water samples are Below Detection Limit (BDL) in some minor and trace elements and other samples are Above Detection Limit (ADL).

6. The isotopic analysis result of the representative groundwater and Nile water samples, as shown in Table 4, has led to the following results: 
Table 3 Minor and trace elements of groundwater samples (values in mg/l)

\begin{tabular}{|c|c|c|c|c|c|c|c|c|c|c|c|c|c|c|c|}
\hline Sample no & Al & B & $\mathrm{Ba}$ & $\mathrm{Cr}$ & $\mathrm{Cu}$ & $\mathrm{Fe}$ & Li & $\mathrm{Mn}$ & Mo & $\mathrm{Ni}$ & $\mathrm{Pb}$ & $\mathrm{Si}$ & Sr & V & Zn \\
\hline Zero & $<0.01$ & $<0.006$ & 0.0259 & $<0.01$ & $<0.006$ & $<0.02$ & 0.0028 & $<0.002$ & $<0.001$ & $<0.002$ & $<0.008$ & 2.007 & 0.232 & $<0.01$ & 0.0009 \\
\hline 1 & .0368 & .0140 & .0361 & .0248 & .0186 & .0499 & .0024 & .0072 & .0046 & $<0.002$ & $<0.008$ & 9.440 & 1.811 & .0108 & .0283 \\
\hline 3 & $<0.01$ & .1490 & .0374 & .0146 & $<0.006$ & $<0.02$ & $<0.0009$ & $<0.002$ & .0078 & $<0.002$ & $<0.008$ & 7.870 & 3.882 & $<0.01$ & $<0.0006$ \\
\hline 5 & $<0.01$ & .1301 & .0511 & $<0.01$ & $<0.006$ & $<0.02$ & $<0.0009$ & .0383 & .0079 & $<0.002$ & $<0.008$ & 8.574 & 2.280 & $<0.01$ & .0047 \\
\hline 7 & .0177 & .1910 & .0639 & $<0.01$ & $<0.006$ & $<0.02$ & $<0.0009$ & $<0.002$ & .0167 & $<0.002$ & $<0.008$ & 7.499 & 6.349 & $<0.01$ & .0007 \\
\hline 8 & $<0.01$ & .0857 & .0349 & $<0.01$ & $<0.006$ & $<0.02$ & $<0.0009$ & $<0.002$ & .0169 & $<0.002$ & $<0.008$ & 7.949 & .7202 & .0107 & $<0.0006$ \\
\hline 11 & $<0.01$ & .0967 & .0903 & .0146 & $<0.006$ & $<0.02$ & .0045 & $<0.002$ & .0038 & $<0.002$ & $<0.008$ & 10.38 & 2.637 & .0321 & $<0.0006$ \\
\hline 15 & $<0.01$ & .0086 & .0219 & $<0.01$ & $<0.006$ & $<0.02$ & $<0.0009$ & $<0.002$ & .0030 & $<0.002$ & $<0.008$ & 9.454 & 1.259 & .0201 & .0052 \\
\hline 18 & $<0.01$ & .0398 & .0315 & .0145 & $<0.006$ & .0956 & .0029 & .0099 & .0041 & $<0.002$ & $<0.008$ & 8.725 & 2.789 & .0211 & $<0.0006$ \\
\hline 19 & $<0.01$ & .3859 & .0449 & .0144 & $<0.006$ & $<0.02$ & .0024 & $<0.002$ & .0060 & $<0.002$ & $<0.008$ & 8.910 & 9.908 & $<0.01$ & $<0.0006$ \\
\hline 24 & .0700 & .0736 & .0588 & $<0.01$ & $<0.006$ & .1183 & $<0.0009$ & .0036 & .0041 & $<0.002$ & $<0.008$ & 5.557 & .4000 & $<0.01$ & .0047 \\
\hline 28 & .0244 & .1178 & .0465 & .0220 & $<0.006$ & $<0.02$ & .0084 & $<0.002$ & .0089 & $<0.002$ & $<0.008$ & 7.702 & 3.143 & .0273 & $<0.0006$ \\
\hline 37 & $<0.01$ & .1937 & .0499 & $<0.01$ & $<0.006$ & $<0.02$ & .0025 & .0337 & .0099 & $<0.002$ & $<0.008$ & 8.890 & 4.666 & $<0.01$ & .0916 \\
\hline 39 & .0213 & .1955 & .0344 & .0634 & .0072 & .0602 & .0495 & $<0.002$ & .0095 & .0045 & .0158 & 13.16 & 3.667 & $<0.01$ & .0175 \\
\hline 40 & .0156 & .1059 & .0563 & $<0.01$ & $<0.006$ & $<0.02$ & .0120 & $<0.002$ & .0297 & $<0.002$ & $<0.008$ & 19.53 & 1.312 & .0986 & $<0.0006$ \\
\hline 42 & $<0.01$ & .1475 & $<0.0004$ & $<0.01$ & .0099 & .1381 & $<0.0009$ & $<0.002$ & .0122 & $<0.002$ & $<0.008$ & 7.959 & 3.341 & $<0.01$ & .0008 \\
\hline 48 & $<0.01$ & .1065 & .0083 & $<0.01$ & $<0.006$ & $<0.02$ & .0034 & .0052 & .0113 & $<0.002$ & $<0.008$ & 8.910 & .5590 & $<0.01$ & .1024 \\
\hline
\end{tabular}

Table 4 Stable isotopes concentration of the represented surface and groundwater samples (Dec. 2016)

\begin{tabular}{lcclcc}
\hline Sample no & $\boldsymbol{\delta}^{\mathbf{1 8}} \mathbf{O} \% \mathbf{o}$ & $\boldsymbol{\delta} \mathbf{D} \% \mathbf{0}$ & Sample no & $\boldsymbol{\delta}^{\mathbf{1 8}} \mathbf{O} \% \mathbf{0}$ & $\boldsymbol{\delta} \mathbf{D} \% \mathbf{o}$ \\
\hline Zero & 4.46 & 32.67 & 19 & -0.30 & -1.14 \\
1 & -1.49 & -8.85 & 24 & -8.66 & -66.48 \\
3 & -2.88 & -26.56 & 37 & -5.73 & -47.45 \\
7 & -1.37 & -28.41 & 39 & 0.91 & 9.09 \\
8 & -1.12 & -11.34 & 40 & 3.35 & 24.03 \\
11 & -0.44 & -2.23 & 42 & -0.34 & -5.96 \\
15 & -1.39 & -7.24 & 48 & -0.53 & -8.40 \\
18 & -0.87 & -7.06 & & & \\
\hline
\end{tabular}

i. The content of oxygen eighteen and deuterium in water samples ranges from -5.73 to $4.46 \%$ o and from -66.48 to $32.67 \%$, respectively.

ii. The representative groundwater samples show the isotope signature of the Nile River sample; this means that all these wells have the same source of recharge (meteoric water of Nile River).

iii. According to the relationship between ${ }^{18} \mathrm{O}$ and D (Table 4 and Fig. 9), almost all groundwater samples belong to the global precipitation with a slight influence of evaporation.

iv. The saline water samples show a high $\delta^{18} \mathrm{O}$ values (more positive $\delta^{18} \mathrm{O}$ ) according to the relationship between TDS and ${ }^{18} \mathrm{O}$ (Fig. 10).

v. All analyzed samples show the isotopic signature of Nile River sample (Sample No zero),

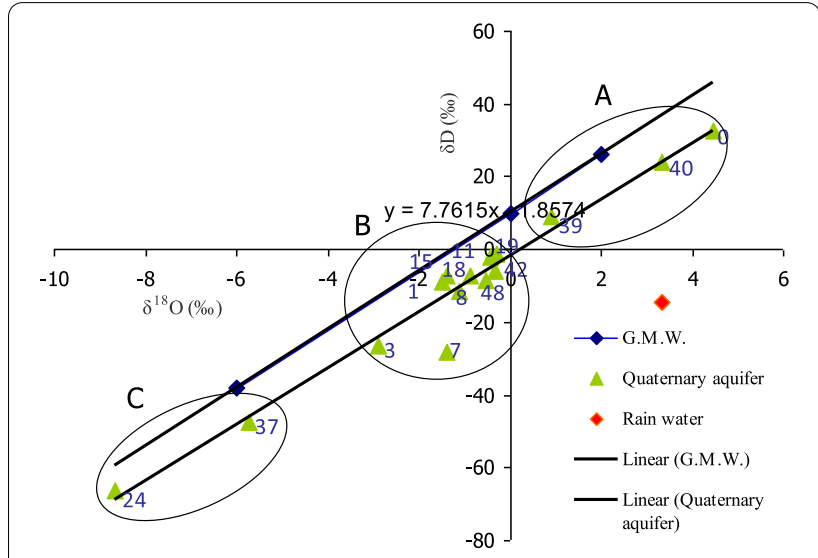

Fig. 9 Relationship between $\delta^{18} \mathrm{O}$ and deuterium ratios of the analyzed samples

which is extremely heavy, due to the intensive evaporation of Nile River which tends to enrich the heavy isotopes in the water, and also found that at samples (No. 39 and 40), which were collected from hand dug wells with low depth to water and exposed to evaporation.

vi. The groundwater isotopic data reflect variability in recharge conditions during different ages and different climatic regimes (secondary fractionation has occurred). Depending on the groundwater local meteoric line, 3 groups can be defined as shown in Fig. 9. Group A characterizes samples that exhibit enrich- 


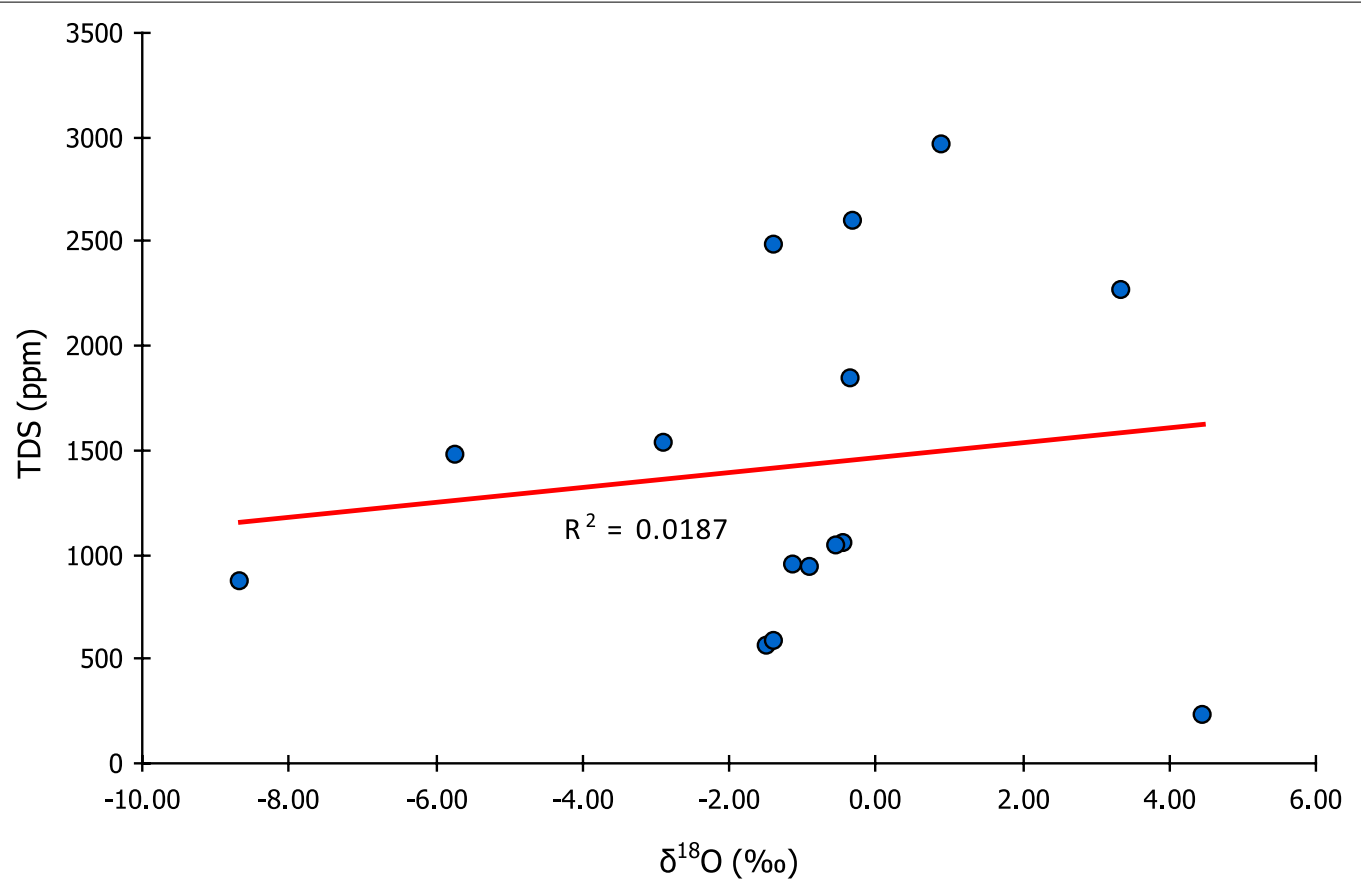

Fig. 10 Relationship between $\delta^{18} \mathrm{O}$ and TDS of the analyzed samples

ment in heavier isotopic composition (positive $\delta 18 \mathrm{O}$ and $\delta \mathrm{D}$ ), due to the intensive evaporation exposed to it, and it includes samples No. (zero, 40 and 39). Group B characterizes samples that display the same recharge conditions (closed basin) affected by local recent recharge events from the surface water bodies ex. canals, drains, Nile River and rain precipitation and includes samples (No 1, 3, 7, 8, 11, 15, 18, 19, 42 and 48). Group C characterizes samples that show light isotopic composition (more negative $\delta \mathrm{D}$ and $\delta 18 \mathrm{O}$ values) and gives an indication that these samples were ancient (paleowater) and originated from rains of a different climatic regime and/or may be recharged from deep aquifers. It includes samples (No. 24 and 37).

vii. The relationship between TDS and ${ }^{18} \mathrm{O}$ (Fig. 10) shows the effect of evaporation as the cause of the rise in salinity with $R=0.0187$. The saline water samples show high $\delta^{18} \mathrm{O}$ values (more positive $\delta^{18} \mathrm{O}$ ). With increasing salinity of ground water samples, it results in the isotopically enriched groundwater.

\section{Discussion}

The evaluation of water samples for irrigation according to the plotting of groundwater samples within the US Salinity Lab. Staff nomogram (1954) revealed the following characters as shown in Fig. 11 and Table 5:

1. Eight percent of the groundwater samples (No. 1, 6, 15 and 33) are located in class $\mathrm{C}_{3}-\mathrm{S}_{1}$ (high salinity and low sodium). The waters of such class can be used for all soils particularly those with moderate to good permeability and leaching while it cannot be used in soil with restricted drainage. Even with adequate drainage, special management for salinity control may be required, and plants with good salt tolerance should be selected.

2. Twenty eight percent of the groundwater samples No. $(4,5,9,11,12,14,17,18,28,30,31,32$ ', 38 and 44) are located in class $\mathrm{C}_{3}-\mathrm{S}_{2}$ (high salinity and medium sodium). The water of such class is preferably used in coarse textured or organic soils with good permeability while it is unsatisfactory for highly clayey soils with low leaching. The waters of this class can be improved by adding gypsum to the soil periodically.

3. Sixteen percent\% of the groundwater samples No. $(3,16,21,23,26,32,34$ and 37$)$ are located in class 
Table 5 Irrigation water classes (According to US Lab. Staff classification 1954)

\begin{tabular}{|c|c|c|c|c|c|c|c|}
\hline Well no & $\begin{array}{l}\text { Ec } \\
(\mu \mathrm{moh})\end{array}$ & SAR & Class & Well no & $\begin{array}{l}\text { Ec } \\
(\mu \mathrm{moh})\end{array}$ & SAR & Class \\
\hline 1 & 1130 & 5.15 & $C_{3}-S_{1}$ & 26 & 4380 & 4.75 & $\mathrm{C}_{4}-\mathrm{S}_{2}$ \\
\hline 2 & 2910 & 11.15 & $\mathrm{C}_{4}-\mathrm{S}_{3}$ & 27 & 4690 & 10.52 & $\mathrm{C}_{4}-\mathrm{S}_{3}$ \\
\hline 3 & 2500 & 6.57 & $\mathrm{C}_{4}-\mathrm{S}_{2}$ & 28 & 1990 & 7.05 & $\mathrm{C}_{3}-\mathrm{S}_{2}$ \\
\hline 4 & 2220 & 7.83 & $\mathrm{C}_{3}-\mathrm{S}_{2}$ & 29 & 1810 & 11.41 & $C_{3}-S_{3}$ \\
\hline 5 & 2200 & 9.11 & $\mathrm{C}_{3}-\mathrm{S}_{2}$ & 30 & 2060 & 5.16 & $C_{3}-S_{2}$ \\
\hline 6 & 2030 & 4.29 & $\mathrm{C}_{3}-\mathrm{S}_{1}$ & 31 & 2110 & 6.11 & $C_{3}-S_{2}$ \\
\hline 7 & 4040 & 8.25 & $\mathrm{C}_{4}-\mathrm{S}_{3}$ & 32 & 3580 & 7.33 & $\mathrm{C}_{4}-\mathrm{S}_{2}$ \\
\hline 8 & 1540 & 12.43 & $\mathrm{C}_{3}-\mathrm{S}_{3}$ & $32^{\prime}$ & 1600 & 5.18 & $C_{3}-S_{2}$ \\
\hline 9 & 2130 & 5.29 & $\mathrm{C}_{3}-\mathrm{S}_{2}$ & 33 & 1270 & 3.19 & $C_{3}-S_{1}$ \\
\hline 10 & 2060 & 11.65 & $\mathrm{C}_{3}-\mathrm{S}_{3}$ & 34 & 2710 & 7.27 & $\mathrm{C}_{4}-\mathrm{S}_{2}$ \\
\hline 11 & 1630 & 6.84 & $\mathrm{C}_{3}-\mathrm{S}_{2}$ & 35 & 2260 & 10.99 & $C_{3}-S_{3}$ \\
\hline 12 & 1780 & 8.07 & $\mathrm{C}_{3}-\mathrm{S}_{2}$ & 36 & 2060 & 9.49 & $\mathrm{C}_{3}-\mathrm{S}_{3}$ \\
\hline $12^{\prime}$ & 2770 & 13.18 & $\mathrm{C}_{4}-\mathrm{S}_{3}$ & 37 & 2390 & 7.57 & $C_{4}-S_{2}$ \\
\hline 13 & 2320 & 9.78 & $\mathrm{C}_{3}-\mathrm{S}_{3}$ & 38 & 1940 & 9.24 & $C_{3}-S_{2}$ \\
\hline 14 & 1570 & 7.09 & $\mathrm{C}_{3}-\mathrm{S}_{2}$ & 39 & 4240 & 13.01 & $\mathrm{C}_{4}-\mathrm{S}_{4}$ \\
\hline 15 & 950 & 4.90 & $C_{3}-S_{1}$ & 40 & 3490 & 15.40 & $C_{4}-S_{4}$ \\
\hline 16 & 2470 & 7.11 & $\mathrm{C}_{4}-\mathrm{S}_{2}$ & 41 & 2990 & 25.17 & $\mathrm{C}_{4}-\mathrm{S}_{4}$ \\
\hline 17 & 2080 & 5.80 & $\mathrm{C}_{3}-\mathrm{S}_{2}$ & 42 & 2630 & 15.71 & $\mathrm{C}_{4}-\mathrm{S}_{4}$ \\
\hline 18 & 1530 & 6.95 & $\mathrm{C}_{3}-\mathrm{S}_{2}$ & 43 & 2030 & 15.27 & $C_{3}-S_{4}$ \\
\hline 19 & 4050 & 7.79 & $\mathrm{C}_{4}-\mathrm{S}_{3}$ & 44 & 2300 & 5.15 & $\mathrm{C}_{3}-\mathrm{S}_{2}$ \\
\hline 20 & 3530 & 8.90 & $\mathrm{C}_{4}-\mathrm{S}_{3}$ & 45 & 4390 & 10.59 & $\mathrm{C}_{4}-\mathrm{S}_{3}$ \\
\hline 21 & 4310 & 6.67 & $\mathrm{C}_{4}-\mathrm{S}_{2}$ & 46 & 4600 & 11.40 & $\mathrm{C}_{4}-\mathrm{S}_{3}$ \\
\hline 22 & 6220 & 9.20 & $C_{4}-S_{3}$ & 47 & 5090 & 12.48 & $C_{4}-S_{4}$ \\
\hline 23 & 2350 & 5.60 & $\mathrm{C}_{4}-\mathrm{S}_{2}$ & 48 & 1770 & 16.59 & $\mathrm{C}_{3}-\mathrm{S}_{4}$ \\
\hline 24 & 1380 & 16.34 & $C_{3}-S_{4}$ & Zero & 350 & 0.89 & $C_{2}-S_{1}$ \\
\hline 25 & 4120 & 8.03 & $\mathrm{C}_{4}-\mathrm{S}_{3}$ & & & & \\
\hline
\end{tabular}

$\mathrm{C}_{4}-\mathrm{S}_{2}$ (very high salinity and medium sodium). Due to high salinity, this water is not suitable for irrigation under ordinary conditions but may be used occasionally under very special circumstances. The soils must be permeable, drainage must be adequate, irrigation water must be applied in excess to provide considerable leaching, and very salt-tolerant crops should be selected.

4. Twelve percent of the groundwater samples No. (8, $10,13,29,35$ and 36) are located in class $\mathrm{C}_{3}-\mathrm{S}_{3}$ (high salinity and high sodium), may produce harmful levels of exchangeable sodium in most soils and will require special soil management good drainage, high leaching, and additions of organic matter, not used in soil with restricted drainage, require chemical amendments. While $20 \%$ of the groundwater samples No. (2, 7, 12, 19, 20, 22, 25, 27, 45 and 46) are located in class $\mathrm{C}_{4}-\mathrm{S}_{3}$ (very high salinity and high sodium), these types cause harmful sodium accumulation in most soils; needs good drainage, high leaching and organic matter addition. Chemical amendments may be required for the replacement of exchangeable sodium, except those amendments may not be feasible with waters of very high salinity.

5. Six percent of the groundwater samples No. $(24,43$ and 48) are located in class $\mathrm{C}_{3}-\mathrm{S}_{4}$ (high salinity and very high sodium). It is generally unsatisfactory for irrigation purposes except at low and perhaps medium salinity where the solution of calcium from the soil or use of gypsum or other amendments may make the use of these waters feasible, while $10 \%$ of the groundwater samples No. (39, 40, 41, 42 and 47) are located in class $\mathrm{C}_{4}-\mathrm{S}_{4}$ (very high salinity and very high sodium). This water is generally unsuitable for irrigation except for high permeable soils with frequent leaching and high tolerant plants. Chemical amendments must be used for exchanging sodium ions from these light sodium-affected soils.

The evaluation of the groundwater for drinking uses in the present work is determined on basis of salinity and minor and trace elements as follow: 


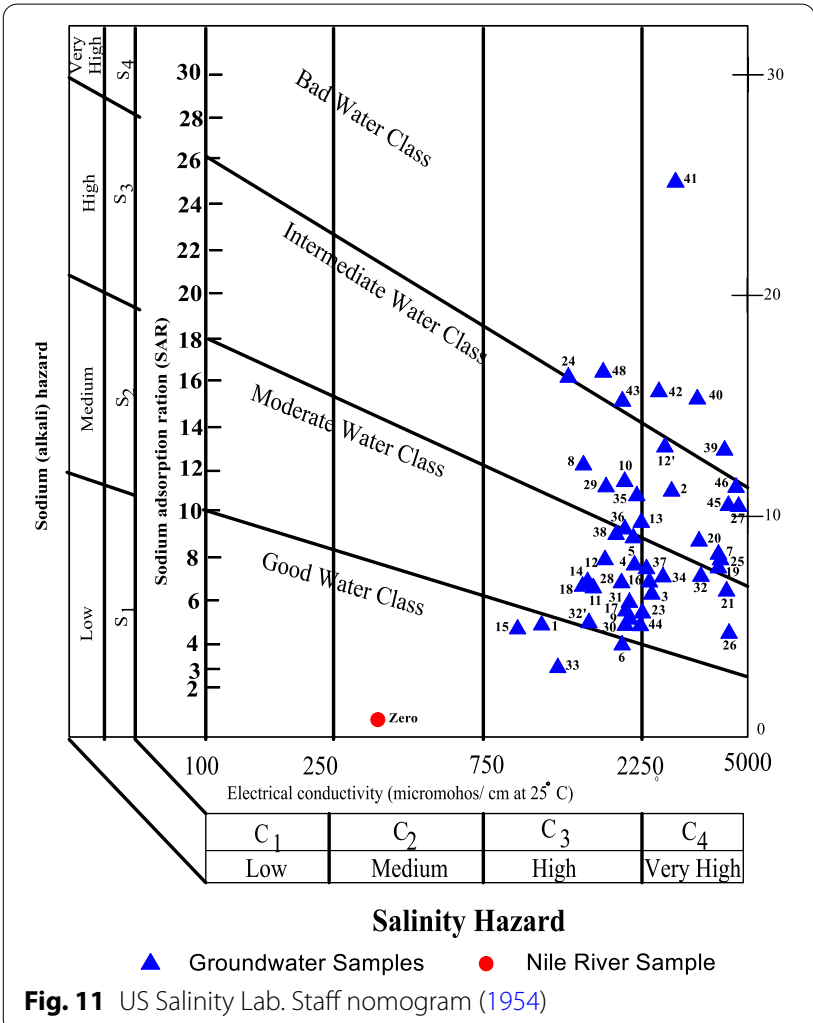

1. According to salinity and minor and trace elements, different references water standards were considered, where it is found that $14 \%$ of the studied samples are acceptable groundwater, $42 \%$ of the samples are permissible and $44 \%$ of the samples are unsuitable groundwater. For minor and trace elements concentration, the analyzed groundwater samples suitable for drinking purposes, except water sample (No. 40), have high silicate content.

\section{Conclusions}

The desert environs west Qena area consists of three types of water-bearing formations, Nubain sandstone, Eocene and the Quaternary aquifer. The Quaternary aquifer represents the most important groundwater aquifer in the area. It is mainly composed of graded sands and gravels. The water in this aquifer is found under semi-confined conditions (under the old cultivated area) and unconfined conditions (under the reclaimed area). It ranges from +40 to $+67 \mathrm{~m}$ above sea level. The groundwater movement direction is mainly from southeast to northwest direction, with some local groundwater flow directions. The lower values of salinity are observed near desert fringes while the higher values are observed along with the young cultivated plain. Sodium and chloride are the main ions constituents of examined wells. The groundwater belongs to one genetic water type, alkaline water with prevailing sulfate and chloride. According to the isotopic analysis result $\left(\delta^{18} \mathrm{O}\right.$ and $\left.\delta^{2} \mathrm{H}\right)$, the representative groundwater samples show the isotope signature of the Nile River sample; this means that all these wells have the same source of recharge (meteoric water of Nile River) with various recharge conditions during different ages and different climatic regimes. Almost all groundwater samples belong to the global precipitation with slightly influence of evaporation. According to the minor and trace elements concentration and US Salinity Lab. Staff nomogram; most of the water samples are suitable for drinking purposes, domestic use and irrigation.

\section{Abbreviations}

TDS: Total dissolved solids; EC: Electrical conductivity; IC: Ion Chromatography; DRC: Desert Research Center; ICP: Inductively coupled plasma; DTW: Depth to water; T.D.: Total depth; G.E.: Ground elevation; W.L.: Absolute water level; BDL: Below detection limit; ADL: Above detection limit.

\section{Acknowledgements}

It is a pleasure to acknowledge Prof. Dr. Michael Schneider (Professor of Hydrogeology, Institute of Geological Science, Freie Universität Berlin) for his cooperation and laboratory facilities were offered during this work.

\section{Authors' contributions}

MAHSES conceived and planned the original idea. MAR and KNS read the first draft of the manuscript for necessary corrections. NHR supervised and contributed to the experimental design and performed analytical computations. OHE carried out the field experiments, collected data for the study and wrote the first draft of the manuscript. All authors wrote, reviewed and approved the final manuscript for submission.

\section{Funding}

No funding was obtained for this study.

\section{Availability of data and materials}

The data used to support the findings of this study are available from the corresponding author upon request.

\section{Declarations}

Ethics approval and consent to participate

The studies involved in this article did not include animals or human participants as objects of research (not applicable).

\section{Consent for publication}

Not applicable.

\section{Competing interests}

The authors declare that they have no conflict of interest.

\section{Author details}

${ }^{1}$ Hydrology Department, Desert Research Center, P.O. Box 11753, El Matariya, Cairo, Egypt. ${ }^{2}$ Geology Department, Faculty of Science, Alexandria University, Alexandria, Egypt.

Received: 14 October 2021 Accepted: 18 January 2022

Published online: 29 January 2022 


\section{References}

Abd El-LatifTA, Abd El-Rahman AA, El-Hefnawy M, Ali MZT (2012) Geoelectrical applications for groundwater exploration in El-Marashda area, Qena. Egypt Assiut Univ J Geol 41(1):87-110

Coral C (1987) Geological map of Egypt, sheets: NG 36 NW Assiut and NG 36 SW Luxor, Scale 1: 500,000. The Egyptian General Petroleum Corporation (EGPC), Cairo

El Sabri MAS (2010) Impact of intensive land reclamation activity on shallow groundwater aquifers in the area between El Wagf and Dandara, west Nile Valley. Qena Assiut Univ J Geol 39(2):57-77

El-Sheikh AE, Barseem MS, Sherbeni WM (2015) Susceptibility of shallow groundwater aquifers to water logging, case study Al Marashda area, West Nile Valley, Qena, Egypt. Mansoura Eng J (MEJ) 40(4):1-8

Mahmoud AM (2005) Hydrogeological and hydrogeochemical evaluation of the area between Qena and Qus, Eastern desert, Egypt. M.Sc. Thesis, Faculty of Science, South Valley University

Piper AM (1944) A graphic procedure in the geochemical interpretation of water analyses. Trans Am Geophys Union 25:914-923

Said R (1962) The geology of egypt. El Sevier, Amsterdam

Said R (1981) The geological evaluation of the River Nile. Springier, New York

Tutiempo Net (2018) https://en.tutiempo.net/climate/Egypt.html

U.S. Salinity Laboratory Staff (1954) Diagnoses and improvement of saline and alkali soils, Handbook No. 60. United States Department of Agriculture, Washington, DC

Youssef M (1968) Structure pattern of Egypt and its interpretation. Am Assoc Petrol Geol Bull 5214:601-614

\section{Publisher's Note}

Springer Nature remains neutral with regard to jurisdictional claims in published maps and institutional affiliations.

\section{Submit your manuscript to a SpringerOpen ${ }^{\circ}$ journal and benefit from:}

- Convenient online submission

- Rigorous peer review

- Open access: articles freely available online

- High visibility within the field

- Retaining the copyright to your article

Submit your next manuscript at $\boldsymbol{\nabla}$ springeropen.com 